DisCRETE ANALYSIS, 2017:7, 37 pp.

www.discreteanalysisjournal.com

\title{
Analyticity of the Entropy and the Escape Rate of Random Walks in Hyperbolic Groups
}

\author{
Sébastien Gouëzel \\ Received 29 September 2015; Revised 19 November 2016; Published 9 May 2017
}

\begin{abstract}
We consider random walks on a nonelementary hyperbolic group endowed with a word distance. To a probability measure on the group are associated two numerical quantities, the rate of escape and the entropy. On the set of admissible probability measures whose support is contained in a given finite set, we show that both quantities depend in an analytic way on the probability measure. Our spectral techniques also give a new proof of the central limit theorem, and imply that the corresponding variance is analytic.
\end{abstract}

\section{Introduction}

Let $\Gamma$ be a finitely generated group. Let $\mu$ be a finitely supported probability measure on $\Gamma$, whose support generates $\Gamma$ as a semigroup (we say that $\mu$ is admissible). It defines a random walk on $\Gamma$, by $Z_{n}=g_{1} \cdots g_{n}$ where $g_{1}, g_{2}, \ldots$ is a sequence of $\Gamma$-valued i.i.d. random variables with distribution $\mu$. By definition, $Z_{n}$ is distributed according to the convolution product $\mu^{* n}$. Equivalently, $Z_{n}$ is a Markov chain on $\Gamma$, starting from the identity $e$ of $\Gamma$, whose transition probabilities are $p(x \rightarrow y)=\mu\left(x^{-1} y\right)$. These probabilities are invariant under left multiplication, hence the Markov chain is homogeneous.

The behavior of the random walk is usually strongly related to the geometric properties of the group. One can associate several numerical quantities to the random walk, including:

- The entropy, defined by

$$
h(\mu)=-\lim _{n \rightarrow \infty} \log \mu^{* n}\left(\left\{Z_{n}\right\}\right) / n .
$$

The almost sure convergence of this quantity follows from Kingman's subadditive theorem. Essentially, the walk at time $n$ visits $e^{h n}$ points. 
- The escape rate, or drift, defined by

$$
\ell(\mu)=\lim _{n \rightarrow \infty} d\left(e, Z_{n}\right) / n,
$$

where $d$ is a fixed (proper, left-invariant) distance on $\Gamma$. Again, the convergence follows from Kingman's theorem.

These quantities are always non-zero in non-amenable groups, and always zero in nilpotent groups for symmetric walks. Our focus in this article will be on the former category, and especially on the subclass of hyperbolic groups.

In hyperbolic groups, Erschler and Kaimanovich have proved in [EK13] that both the entropy and the rate of escape depend continuously on the measure. If one concentrates on measures with a given finite support, then the parameter space becomes a subset of $\mathbb{R}^{d}$ for some $d$, and one can investigate further regularity properties. Our goal in this article is to show that the entropy, and the rate of escape for a word distance, are analytic.

Let $\Gamma$ be a nonelementary hyperbolic group, endowed with a word distance $d$. Let $F$ be a finite subset of $\Gamma$. Denote by $\mathcal{P}_{1}^{+}(F)$ the set of admissible probability measures supported by $F$ : this is a subset of the finite-dimensional space $\mathcal{P}(F)=\{\mu: F \rightarrow \mathbb{C}\}$. Our main theorem is the following.

Theorem 1.1. In this setting, the functions $\mu \mapsto \ell(\mu)$ and $\mu \mapsto h(\mu)$ are analytic on $\mathcal{P}_{1}^{+}(F)$. More precisely, there exists an open subset of $\mathcal{P}(F)$ containing $\mathcal{P}_{1}^{+}(F)$ on which these two functions extend to analytic functions.

The random walk converges almost surely to a point of the Gromov boundary of $\Gamma$. The distribution of the limit is called the exit measure of the random walk. Its Hausdorff dimension is proportional to $h / \ell$ [Haï13, Theorem 4.11]. Thus, it follows from the previous theorem that it also depends analytically on $\mu$.

This theorem extends several partial results in the literature. Here are some previous results in this direction, we refer the reader to [GL13a] for more statements.

- For nearest neighbor random walks on the free group, one can obtain explicit formulas for $h(\mu)$ and $\ell(\mu)$, thereby proving analyticity. Similar results hold in free products, see [Gil07, Gil11].

- In a free group, for measures with a given finite support, both the rate of escape and the entropy are analytic, see respectively [Gil08] and [Led12].

- In a general hyperbolic group, but for a restricted class of distances, Gilch and Ledrappier prove in [GL13a] that the rate of escape is analytic. The condition on the distance is that its Busemann boundary should coincide with the Gromov boundary (these terms are defined in Paragraph 2.1). This is for instance the case if $\Gamma$ acts cocompactly by isometries on a hyperbolic space $\mathbb{H}^{n}$ and the distance is given by $d\left(g, g^{\prime}\right)=d_{\mathbb{H}^{n}}\left(g \cdot O, g^{\prime} \cdot O\right)$ where $O$ is a suitable basepoint. On the other hand, if $\Gamma$ is not virtually free, this is never the case for a word distance as the Busemann boundary is totally disconnected, contrary to the Gromov boundary (see Paragraph 2.1).

- In surface groups, [HMM16] shows that the rate of escape is analytic. 


\section{ANALYTICITY OF THE ENTROPY AND THE EsCAPE RATE}

- In a general hyperbolic group, Ledrappier proves in [Led13] that both the rate of escape and the entropy are Lipschitz continuous.

- This is improved to $C^{1}$ by Mathieu in [Mat15].

Our approach to prove Theorem 1.1 is based on the strategy of Ledrappier in [Led13]: We express the rate of escape and the entropy as integrals of objects living on a suitable boundary of the group, the Busemann boundary $\partial_{B} \Gamma$. What we have to show is that these objects (the stationary measure and the Martin kernel) depend analytically on the measure $\mu$.

More precisely, let $v_{\mu}$ be a stationary measure for $\mu$ on $\partial_{B} \Gamma$. Let $K_{\mu}$ be the Martin kernel for $\mu$, and $K_{\mu}^{B}$ its lift to $\partial_{B} \Gamma$. Let $c_{B}$ be the Busemann cocycle on $\partial_{B} \Gamma$. (All these terms will be defined in Paragraph 2.1.) Then the following formulas are classical (see for instance [GMM15, Proposition 2.2] for (1.1), and [KV83, Theorem 3.1] or the comments before (2.10) for (1.2)):

$$
\ell(\mu)=\sum_{g \in \Gamma} \mu(g) \int_{\partial_{B} \Gamma} c_{B}(g, \xi) \mathrm{d} v_{\mu}(\xi)
$$

and

$$
h(\mu)=-\sum_{g \in \Gamma} \mu(g) \int_{\partial_{B} \Gamma} \log K_{\mu}^{B}\left(g^{-1}, \xi\right) \mathrm{d} v_{\mu}(\xi) .
$$

There are two main difficulties to prove analyticity statements using these formulas.

First, to use (1.1), we need to know that $\mu \mapsto v_{\mu}$ is analytic in some sense. This is well known if the action of $\Gamma$ on $\partial_{B} \Gamma$ has contraction properties, for instance if $\partial_{B} \Gamma$ coincides with the Gromov boundary $\partial \Gamma$. However, if $\Gamma$ is not virtually free, the projection $\pi_{B}: \partial_{B} \Gamma \rightarrow \partial \Gamma$ is not one-to-one (see Paragraph 2.1), and points in the same fiber of $\pi_{B}$ do not feel contraction. As a consequence, we do not know if there is a unique stationary measure on $\partial_{B} \Gamma$. To work around this problem, we work on a subset $\partial_{B}^{\prime} \Gamma$ of $\partial_{B} \Gamma$ which is minimal for the $\Gamma$-action, and show using a non-constructive spectral argument that there is some form of contraction there (more precisely, a spectral gap for the Markov operator associated to the walk, on a suitable function space, with a simple dominating eigenvalue). Hence, there is a unique stationary measure on $\partial_{B}^{\prime} \Gamma$, depending analytically on $\mu$. Together with (1.1), this shows that $\ell(\mu)$ depends analytically on $\mu$.

Second, in (1.2), the cocycle $\log K_{\mu}^{B}\left(g^{-1}, \xi\right)$ also depends on $\mu$. This difficulty is serious, making the entropy harder to study in general than the escape rate. To get the analyticity of the entropy, we need to show that $\mu \mapsto K_{\mu}^{B}$ is analytic in some sense. Ledrappier obtains in [Led13] the kernel $K_{\mu}^{B}(\cdot, \xi)$ for each $\xi$ by applying a sequence of contracting operators depending on $\xi$. Choosing carefully the number of contraction steps, he deduces a Lipschitz control on $K_{\mu}^{B}$, and thus that the entropy is Lipschitz. Instead, we will exhibit the whole kernel $(x, \xi) \mapsto K_{\mu}^{B}(x, \xi)$ as the unique fixed point of a non-linear operator, depending analytically on $\mu$. The analyticity of $\mu \mapsto K_{\mu}^{B}$ then follows from a suitable application of the implicit function theorem.

This approach is reminiscent of the study of unstable foliations in hyperbolic dynamics. The usual strategy is to apply a map called the graph transform, enjoying contraction properties, to obtain the unstable leaf at a point. Unstable leaves at nearby points are then compared by iterating the graph transform a finite but carefully chosen number of times. Another approach, advocated by Hirsch, Pugh and Shub in [HPS77], is to see the whole family of unstable manifolds as the fixed point of a single 


\section{SÉBASTIEN GOUËZEL}

operator. The proper setting is more complicated to develop, but once this is done it is extremely powerful. We follow essentially a similar strategy.

Remark 1.2. The kernel $K_{\mu}(x, \xi)$ is initially defined on the (geometric) Gromov boundary. However, to construct an operator of which it is a fixed point, it is convenient to have an underlying combinatorial structure. This is more efficiently done using the Busemann boundary. Therefore, although the statement on analyticity on the entropy does not depend on a distance choice, its proof relies on the tools we develop to show that the escape rate is analytic for the word distance.

Remark 1.3. The escape rate $\ell(\mu)$ depends on the measure $\mu$, but also on the choice of a distance on $\Gamma$. Theorem 1.1 is formulated for a word distance. Other natural distances can be constructed as follows. Consider a free cocompact action of $\Gamma$ by isometries on a Gromov-hyperbolic space $X$, take a basepoint $O \in X$, and define $d\left(g, g^{\prime}\right)=d_{X}\left(g \cdot O, g^{\prime} \cdot O\right)$. (The case of a word distance corresponds to the natural action of $\Gamma$ on its Cayley graph with the graph distance). We do not know if the escape rate $\ell(\mu)$ depends analytically on $\mu$ in this generality. Indeed, our proofs rely on some contraction properties of the dynamics on the Busemann boundary, for some suitable distance, which are not clear in this generality.

For the word distance, we prove below that these requirements are met (the distance on the Busemann boundary is defined in (2.2), the contraction properties are proved in Lemma 3.5). The situation is also well behaved if one considers an action of $\Gamma$ by isometries on $X=\mathbb{H}^{k}$ (or a more general $C A T(-1)$ space) and defines a distance $d$ on $\Gamma$ using the $X$ distance on an orbit as above. Indeed, in this case, the Busemann boundary can be identified with a subset of the Gromov boundary of $X$. One thus gets a distance with good contracting properties on the Busemann boundary, from which one can deduce that the drift is analytic following (simplified versions of) the proofs below. This case is in fact easier than the case of the word distance, and was already known, see Corollary 4.2 in [GL13a].

Remark 1.4. Theorem 1.1 only proves analyticity of the escape rate and the entropy on the set of admissible measures. One may hope that these quantities are analytic on a larger set of measures, for instance those that generate a nonelementary subgroup. However, there are considerable difficulties to extend the proofs to this more general situation. For the escape rate, the problem is in Proposition 3.6: we consider there the dominating eigenvalue of an operator $L_{\mu}$ associated to $\mu$. If $\mu$ is not admissible, this eigenvalue can have a nontrivial multiplicity, and perturbations of non-simple eigenvalues can be non-analytic. The situation is even worse for the analyticity of the entropy: there, we make use of the whole machinery of Ancona inequalities (see for instance Proposition 4.2), which are only known for admissible measures.

Once the spectral gap is available, standard techniques due to Le Page [LP82] imply several results on the behavior of the random walk, including the central limit theorem, the law of the iterated logarithm and large deviations estimates. As an illustration, we prove the following statement:

Theorem 1.5. Under the assumptions of Theorem 1.1, there exists for all $\mu \in \mathcal{P}_{1}^{+}(F)$ a real number $\sigma^{2}(\mu)>0$ such that $\left(d\left(e, Z_{n}\right)-n \ell(\mu)\right) / \sqrt{n}$ converges in distribution to $\mathcal{N}\left(0, \sigma^{2}(\mu)\right)$ when $n$ tends to infinity. Moreover, $\mu \mapsto \sigma^{2}(\mu)$ is analytic on $\mathcal{P}_{1}^{+}(F)$.

The central limit theorem is already known under much weaker assumptions on the measure $\mu$ : it suffices that it has a second moment, it does not need to be admissible, and the distance can be 
more general than a word distance. This is proved in [BQ16]. Another approach (which works also in acylindrically hyperbolic groups) has recently been developed by Mathieu and Sisto [MS16]. If one replaces the word distance by a nicer distance (for which the Gromov boundary and the Busemann boundary coincide), then the central limit theorem had been proved earlier by Björklund in [Bjö10].

The analyticity of $\mu \mapsto \sigma^{2}(\mu)$ is proved in [HMM16] for surface groups, but it is new in this generality.

Remark 1.6. The assumption of finite support can be somewhat weakened for the analyticity of $\mu \mapsto$ $\ell(\mu)$ and $\mu \mapsto \sigma^{2}(\mu)$. Indeed, it can be replaced by an exponential moment condition, of the form $\sum|\mu(g)| e^{\alpha|g|}<\infty$, for any fixed $\alpha>0$. Denote by $\mathcal{P}(\alpha)$ the set of measures satisfying this condition (it is a Banach space for the corresponding norm), by $\mathcal{P}_{1}(\alpha)$ its elements with $\sum \mu(g)=1$, and by $\mathcal{P}_{1}^{+}(\alpha)$ its elements which, furthermore, are nonnegative and admissible. Then $\ell$ and $\sigma^{2}$ are analytic on $\mathcal{P}_{1}^{+}(\alpha)$, i.e., they extend to analytic functions on a neighborhood of this set in $\mathcal{P}(\alpha)$. The proofs are exactly the same as for the finite support case, modulo some details on analytic functions on Banach spaces which are explained in Paragraph 2.3.

On the other hand, for the entropy, finite support is essential to our argument. It is likely that it can be weakened to exponential moment of some high enough order, using the techniques of [Gou15], at the price of significant technical complications.

The paper is organized as follows: Section 2 recalls classical results for random walks on hyperbolic groups. Then, the analyticity of the rate of escape is proved in Section 3, and the analyticity of the entropy is proved in Section 4. Finally, Section 5 is devoted to the central limit theorem.

\section{Preliminaries}

In this paragraph, we recall classical results on random walks on hyperbolic groups that we will need later on. See [GdlH90] for a general introduction to hyperbolic groups, and [Led13, Haï13, GMM15] for properties of random walks there.

\subsection{Hyperbolic groups}

Let $\Gamma$ be a finitely generated group, with a finite symmetric generating set $S$. The word distance $d=d_{S}$ is given by

$$
d(x, y)=\inf \left\{n \in \mathbb{N}: \exists s_{1}, \ldots, s_{n} \in S \text { with } x^{-1} y=s_{1} \cdots s_{n}\right\} .
$$

This is the graph distance on the Cayley graph of $(\Gamma, S)$.

The group $\Gamma$ is hyperbolic if all geodesic triangles in its Cayley graph are thin, i.e., there exists $\delta \geqslant 0$ such that each side of such a triangle is included in the $\delta$-neighborhood of the union of the two other sides. This geometric definition has a metric counterpart, as follows. For $x, y, z \in \Gamma$, define the Gromov product of $x$ and $y$ with basepoint $z$ as

$$
(x \mid y)_{z}=(d(x, z)+d(y, z)-d(x, y)) / 2 .
$$


It is small if $z$ is close to a geodesic from $x$ to $y$. One should think of $(x \mid y)_{z}$ as the time during which two geodesics from $z$ to $x$ and from $z$ to $y$ remain close. The group $\Gamma$ is hyperbolic if and only, for some $\delta \geqslant 0$, and for all $x_{1}, x_{2}, x_{3}, z \in \Gamma$,

$$
\left(x_{1} \mid x_{3}\right)_{z} \geqslant \min \left(\left(x_{1} \mid x_{2}\right)_{z},\left(x_{2} \mid x_{3}\right)_{z}\right)-\delta .
$$

A hyperbolic group $\Gamma$ is nonelementary if it is not finite nor virtually $\mathbb{Z}$. Equivalently, it is non-amenable. Then it contains a free group on two generators.

The Gromov boundary of $\Gamma$, denoted by $\partial \Gamma$, is the set of equivalence classes of geodesic rays, where two such rays are equivalent if they stay a bounded distance away. It has a canonical topology, for which $\Gamma \cup \partial \Gamma$ is a compact space. A sequence $x_{n} \in \Gamma$ converges to some point on the boundary if and only if $\left(x_{n} \mid x_{m}\right)_{z}$ tends to infinity when $n, m \rightarrow \infty$ for some, or equivalently all, basepoint $z$.

The group $\Gamma$ acts on itself by left-multiplication. This action extends to a continuous action on $\Gamma \cup \partial \Gamma$, and in particular on $\partial \Gamma$.

All the notions we have described up to now are invariant under quasi-isometry. Hence, they do not depend on the choice of the generating set $S$ or the word metric $d$ (although the precise value of $\delta$ does). We turn to a notion that depends on finer details of the distance (this is necessary to compute the escape rate, which really depends on the distance).

To $y \in \Gamma$, we associate the corresponding normalized distance function $h_{y}(x)=d(x, y)-d(e, y)$. It is 1-Lipschitz and satisfies $h_{y}(e)=0$. A horofunction $h$ on $\Gamma$ is a pointwise limit of a sequence $h_{y_{n}}$ where $y_{n} \rightarrow \infty$. It is still 1-Lipschitz, satisfies $h(e)=0$, and $h(\Gamma) \subseteq \mathbb{Z}$. The map $x \mapsto h_{x}$ is an embedding $\Gamma \rightarrow C^{0}(\Gamma, \mathbb{R})$. Identifying $\Gamma$ with its image, and taking its closure in $C^{0}(\Gamma, \mathbb{R})$ for the topology of pointwise convergence, we obtain a compactification $\Gamma \cup \partial_{B} \Gamma$, where each $\xi \in \partial_{B} \Gamma$ corresponds to a horofunction $h_{\xi}$ obtained as above. The compact space $\partial_{B} \Gamma$ is called the Busemann boundary associated to $(\Gamma, d)$. It really depends on $d$, not just its quasi-isometry class.

If a sequence $y_{n} \in \Gamma$ converges in the Busemann compactification, it also converges in the Gromov compactification. Hence, there is a canonical continuous projection $\pi_{B}: \partial_{B} \Gamma \rightarrow \partial \Gamma$, which is onto but not one-to-one in general. Indeed, the space $\partial_{B} \Gamma$ is made of $\mathbb{Z}$-valued functions, hence it is totally discontinuous, while $\partial \Gamma$ is totally discontinuous if and only if $\Gamma$ is virtually free, see [KB02, Theorem 8.1]. The projection $\pi_{B}$ is uniformly finite-to-one by [CP01].

The left-action of $\Gamma$ on itself extends to a continuous action on $\Gamma \cup \partial_{B} \Gamma$, and in particular on $\partial_{B} \Gamma$. In terms of horofunctions, it is given by the following formula:

$$
h_{g \cdot \xi}(x)=h_{\xi}\left(g^{-1} x\right)-h_{\xi}\left(g^{-1}\right),
$$

where the term $-h_{\xi}\left(g^{-1}\right)$ ensures that the expression on the right vanishes for $x=e$, as it should.

We define a distance on the Busemann boundary by

$$
d\left(\xi, \xi^{\prime}\right)=e^{-n}
$$

where $n$ is the largest number such that the functions $h_{\xi}$ and $h_{\xi^{\prime}}$ coincide on the ball $B(e, n)$. It is compatible with the topology of $\partial_{B} \Gamma$.

Consider a point $\xi \in \partial_{B} \Gamma$, and a subset $A \subseteq \Gamma$ such that $\pi_{B}(\xi)$ does not belong to the closure of $A$ in the Gromov compactification. Then there exists $C$ such that, for all $x \in A$,

$$
\left|d(e, x)-h_{\xi}(x)\right| \leqslant C
$$


Indeed, if $y_{n}$ tends to $\xi$, then for large enough $n$ a geodesic from $x \in A$ to $y_{n}$ intersects a fixed large enough ball around $e$. This implies that $d(e, x)-h_{y_{n}}(x)$ remains uniformly bounded. Letting $n$ tend to infinity, we obtain the claim.

\subsection{Random walks on hyperbolic groups}

Let $\mu$ be an admissible probability measure on a nonelementary hyperbolic group $\Gamma$, endowed with a word distance $d$. Almost surely, trajectories $Z_{n}$ of the corresponding random walk converge in the Gromov compactification $\Gamma \cup \partial \Gamma$, towards a random point $Z_{\infty} \in \partial \Gamma$ [Kai00, Theorem 7.6]. The distribution $v$ of $Z_{\infty}$ is a measure on $\partial \Gamma$, called the exit measure, or hitting measure, or harmonic measure.

An important property of $v$ is that it is $\mu$-stationary, i.e., $\mu * v=v$, i.e.,

$$
v=\sum_{g \in \Gamma} \mu(g) g_{*} \nu .
$$

Indeed, consider the first jump $g$ of the random walk, distributed according to $\mu$, and then the subsequent trajectories starting from $g$. By homogeneity, they are distributed like $g Z_{n-1}$, hence their exit distribution is $g_{*} V$. Averaging with respect to $g$, one obtains (2.4).

Using the contraction properties of the $\Gamma$-action on $\partial \Gamma$, one checks that $v$ is the unique $\mu$-stationary measure on $\partial \Gamma$ [Kai00, Theorem 7.6]. Moreover, it is atomless and has full support.

On the other hand, the random walk does not always converge in the Busemann compactification $\Gamma \cup \partial_{B} \Gamma$. For instance, if $\Gamma=F_{d} \times \mathbb{Z} / 2 \mathbb{Z}$ (where $F_{d}$ is a free group), then the Busemann boundary is made of two copies of $\partial F_{d}$, corresponding to the two elements of $\mathbb{Z} / 2 \mathbb{Z}$. As the random walk keeps jumping between the two sheets $F_{d} \times\{0\}$ and $F_{d} \times\{1\}$, it does not converge.

Nevertheless, by compactness of $\partial_{B} \Gamma$, there are stationary measures on $\partial_{B} \Gamma$. Such measures project under $\pi_{B}$ to the unique stationary measure $v$ on $\partial \Gamma$. Since the projection $\pi_{B}$ is uniformly finite-to-one, there are finitely many ergodic stationary measures on $\partial_{B} \Gamma$, their number being bounded by the cardinality of the fibers. Indeed, the disintegrations above $v$ of such ergodic measures give rise to mutually singular measures on the fibers, and there are at most $N$ mutually singular measures on a space of cardinality $N$. Note however that there is no known example of a hyperbolic group with an admissible measure for which there are more than one stationary measure on $\partial_{B} \Gamma$.

One interest of these stationary measures is that they make it possible to express the escape rate of the random walk. From this point on, we assume that $\mu$ has a moment of order 1. Define the Busemann cocycle

$$
c_{B}: \Gamma \times \partial_{B} \Gamma \rightarrow \mathbb{R}, \quad c_{B}(g, \xi)=h_{\xi}\left(g^{-1}\right) .
$$

It is an (action) cocycle, i.e., it satisfies the following equation:

$$
c_{B}\left(g_{1} g_{2}, \xi\right)=c_{B}\left(g_{1}, g_{2} \xi\right)+c_{B}\left(g_{2}, \xi\right) \text {. }
$$

Moreover, if $v_{B}$ is any stationary probability measure on $\partial_{B} \Gamma$, one has

$$
\ell(\mu)=\int_{\Gamma \times \partial_{B} \Gamma} c_{B}(g, \xi) \mathrm{d} \mu(g) \mathrm{d} v_{B}(\xi)
$$


see for instance [GMM15, Proposition 2.2].

Assume now that $\mu$ has finite support. The Green function associated to $\mu$ is defined by

$$
G_{\mu}(x, y)=\sum_{n} \mu^{* n}\left(x^{-1} y\right) .
$$

It is the average time that the walk started from $x$ spends at $y$. In general, the convergence of this series has a simple probabilistic interpretation, the transience of the random walk. The Green function is also equal to $\sum_{n}\left(Q_{\mu}^{n} \delta_{y}\right)(x)$, where $Q_{\mu}$ is the Markov operator of the random walk, given by $Q_{\mu} f(x)=\sum \mu(g) f(x g)$. Since $\Gamma$ is non-amenable, the spectral radius of this operator acting on $\ell^{2}(\Gamma)$ is $<1$. Hence, the series (2.8) is well defined as its general term tends to 0 exponentially fast. Moreover, if $\mu^{\prime}$ is any measure close enough to $\mu$, then this series still makes sense, even if $\mu^{\prime}$ is not a probability measure any more.

One interest of the Green function is that it is harmonic away from the diagonal: if $x \neq y$, then $G_{\mu}(x, y)=\sum_{g} \mu(g) G_{\mu}(x g, y)$. This follows by considering the first jump of the random walk, from $x$ to $x g$, and then the trajectories from $x g$ to $y$.

We will need some results on the Martin boundary of random walks on groups, as explained for instance in [Saw97]. One says that a sequence $y_{n} \in \Gamma$ converges in the Martin boundary of $(\Gamma, \mu)$ if $G_{\mu}\left(x, y_{n}\right) / G\left(e, y_{n}\right)$ converges to a limit for all $x \in \Gamma$. This defines a compactification of $\Gamma$ by its Martin boundary $\partial_{M} \Gamma$, a compact topological space. By definition, when $y_{n}$ converges to a point $\xi$ in the Martin boundary, then $G_{\mu}\left(x, y_{n}\right) / G\left(e, y_{n}\right)$ converges to a limit denoted by $K_{\mu}(x, \xi)$, the Martin kernel associated to $\xi$. Moreover, the Martin kernel depends continuously on $\xi \in \partial_{M} \Gamma$. A remarkable property is that almost every trajectory of the random walk converges in the Martin boundary. The distribution of the limit is a stationary measure $v_{M}$ on $\partial_{M} \Gamma$. Moreover, the entropy satisfies the following formula:

$$
h(\mu)=-\int_{\Gamma \times \partial_{M} \Gamma} \log K_{\mu}\left(x^{-1}, \xi\right) \mathrm{d} \mu(x) \mathrm{d} v_{M}(\xi) .
$$

This follows from the fact that $\left(\partial_{M} \Gamma, v_{M}\right)$ is a realization of the Poisson boundary of the random walk, from the general formula $K_{\mu}(x, \xi)=\frac{\mathrm{d}\left(x_{*} v_{M}\right)}{\mathrm{d} v_{M}}(\xi)$ on the Martin boundary, and from the integral formula for the entropy on the Poisson boundary given in [KV83, Theorem 3.1]. Since we will not need these notions in the remainder of the paper, readers may take the formula (2.9) as a black box.

In general, it is very difficult to describe explicitly the Martin boundary, i.e., to understand precisely for which sequences $y_{n}$ one has for all $x$ the convergence of $G_{\mu}\left(x, y_{n}\right) / G\left(e, y_{n}\right)$. In the specific case we are considering, i.e., an admissible finitely supported measure on a hyperbolic group, Ancona has proved in [Anc88] that the Martin boundary is exactly the Gromov boundary (and they coincide as topological spaces). In other words, when $y_{n} \in \Gamma$ tends to $\xi \in \partial \Gamma$, then $G_{\mu}\left(x, y_{n}\right) / G\left(e, y_{n}\right)$ converges to a limit $K_{\mu}(x, \xi)$, which is harmonic in $x$ and depends continuously on $\xi$. Thus, the formula (2.9) reads

$$
h(\mu)=-\int_{\Gamma \times \partial \Gamma} \log K_{\mu}\left(x^{-1}, \xi\right) \mathrm{d} \mu(x) \mathrm{d} \nu(\xi),
$$

where $v$ is the unique stationary measure on $\partial \Gamma$ (it coincides with $v_{M}$ by uniqueness).

We will rather use this formula on the Busemann boundary. The Martin kernel lifts to $\Gamma \times \partial_{B} \Gamma$ by the formula $K_{\mu}^{B}(x, \xi)=K_{\mu}\left(x, \pi_{B}(\xi)\right)$. The projection $\left(\pi_{B}\right)_{*} v_{B}$ of any stationary measure $v_{B}$ on $\partial_{B} \Gamma$ is equal 
to $v$. Hence, the previous formula yields

$$
h(\mu)=-\int_{\Gamma \times \partial_{B} \Gamma} \log K_{\mu}^{B}\left(x^{-1}, \xi\right) \mathrm{d} \mu(x) \mathrm{d} v_{B}(\xi) .
$$

\subsection{Analytic maps between Banach spaces}

To prove the analyticity of $\ell$ and $h$, we need to use analytic mappings between Banach spaces. Although this is standard, we recall some details for the convenience of the reader. An excellent reference is [Muj86].

Let $E, F$ be real Banach spaces. A mapping $f$ from an open subset $U$ of $E$ to $F$ is real-analytic (or simply analytic) if, for every $a \in U, f$ has a Taylor expansion around $a$, of the form $f(x)=\sum_{m} P^{m} f(a)(x-$ $a$ ), where $P^{m} f(a)$ is a homogeneous polynomial of degree $m$, i.e., a map of the form $y \mapsto A(y, \ldots, y)$ where $A$ is a continuous $m$-linear map from $E^{m}$ to $F$. We require that the above series converges uniformly on some ball around $a$, i.e., $\sum\left\|P^{m} f(a)\right\| r^{m}<\infty$ for some $r>0$.

When the same property is satisfied in complex Banach spaces, we say that $f$ is complex-analytic, or analytic, or holomorphic.

Let $f: U \subseteq E \rightarrow F$ be a real-analytic map between real Banach spaces. Using its Taylor series, one obtains an extension $f_{\mathbb{C}}$ of $f$ where $f_{\mathbb{C}}: U_{\mathbb{C}} \subseteq E_{\mathbb{C}} \rightarrow F_{\mathbb{C}}$ is holomorphic on a domain $U_{\mathbb{C}}$ of the complexification $E_{\mathbb{C}}=E \otimes \mathbb{C}$, with $U \subseteq U_{\mathbb{C}}$. One can choose to work either with $f$ or $f_{\mathbb{C}}$, i.e., in real or complex Banach spaces. However, holomorphic mappings enjoy several remarkable properties that are not satisfied in the real case, hence we will mainly use the complex point of view. Notably:

- A mapping $f$ is holomorphic if and only if it is everywhere differentiable over $\mathbb{C}$ (i.e., it is $\mathbb{R}$ differentiable and its differential is $\mathbb{C}$-linear) [Muj86, Theorem 13.16]. For instance, this implies without any computation that a composition of holomorphic maps is still holomorphic.

- A mapping $f: U_{\mathbb{C}} \subseteq E_{\mathbb{C}} \rightarrow F_{\mathbb{C}}$ is holomorphic if and only if it is continuous and, for every $a, b \in E_{\mathbb{C}}$, the map $z \mapsto f(a+b z)$ from an open subset of $\mathbb{C}$ to $F_{\mathbb{C}}$ is holomorphic where defined [Muj86, Theorem 8.7].

- A mapping $f: U_{\mathbb{C}} \subseteq E_{\mathbb{C}} \rightarrow F_{\mathbb{C}}$ is holomorphic if and only if it is continuous and, for every $a, b \in E_{\mathbb{C}}$ and every $\psi \in F_{\mathbb{C}}^{\prime}$, the map $z \mapsto \psi \circ f(a+b z)$ from an open subset of $\mathbb{C}$ to $\mathbb{C}$ is holomorphic where defined [Muj86, Theorem 8.12].

- A locally uniform pointwise limit of holomorphic mappings is still holomorphic, by [Muj86, Proposition 9.13].

\section{Analyticity of the escape rate}

Let $\Gamma$ be a nonelementary $\delta$-hyperbolic group, endowed with a word distance. Increasing $\delta$ if necessary, we may assume that it is an integer.

In this section, we prove that the map $\mu \mapsto \ell(\mu)$ is analytic on $\mathcal{P}_{1}^{+}(F)$, as stated in Theorem 1.1. We will even prove the stronger statement given in Remark 1.6. Let $\alpha>0$. Denote by $\mathcal{P}(\alpha)$ the complex 
Banach space of measures $\mu$ such that $\sum|\mu(g)| e^{\alpha|g|}<\infty$, with the corresponding norm $\|\mu\|_{\alpha}$. We will show that $\mu \mapsto \ell(\mu)$ is analytic on a neighborhood of the set $\mathcal{P}_{1}^{+}(\alpha) \subseteq \mathcal{P}(\alpha)$ of admissible probability measures.

The idea is to use (2.7). Thus, we need to exhibit a family of stationary probability measures on the Busemann boundary $\partial_{B} \Gamma$, depending analytically on $\mu$.

We recall that we have defined a distance $d$ on $\partial_{B} \Gamma$ in (2.2). We write $C^{\beta}$ for the space of $\beta$-Hölder continuous function on $\left(\partial_{B} \Gamma, d\right)$, with the norm

$$
\|u\|_{C^{\beta}}=\|u\|_{C^{0}}+\sup _{\xi \neq \xi^{\prime}}\left|u(\xi)-u\left(\xi^{\prime}\right)\right| / d\left(\xi, \xi^{\prime}\right)^{\beta}
$$

The main result of this section is the following.

Theorem 3.1. Let $\alpha>0$ and $\beta>0$. Let $\mu_{0} \in \mathcal{P}_{1}^{+}(\alpha)$ be an admissible probability measure with a finite moment of order $\alpha$. Then there exist a neighborhood $U$ of $\mu_{0}$ in $\mathcal{P}(\alpha)$ and an analytic map $\Phi: U \rightarrow\left(C^{\beta}\right)^{*}$ such that, for $\mu \in U \cap \mathcal{P}_{1}^{+}(\alpha)$, the linear form $\Phi(\mu)$ is given by the integration against a $\mu$-stationary measure on $\partial_{B} \Gamma$.

Before proving this theorem, let us see how it implies the analyticity of the escape rate.

Corollary 3.2. The map $u \mapsto \ell(\mu)$, associating to $\mu \in \mathcal{P}_{1}^{+}(\alpha)$ its escape rate, extends to an analytic map on a neighborhood in $\mathcal{P}(\alpha)$ of any $\mu_{0} \in \mathcal{P}_{1}^{+}(\alpha)$.

Proof. Take $\beta<\alpha$. Let $\Phi(\mu)$ be the functional constructed in Theorem 3.1, on a neighborhood $U \subseteq \mathcal{P}(\alpha)$ of $\mu_{0} \in \mathcal{P}_{1}^{+}(\alpha)$. For $\mu \in U \cap \mathcal{P}_{1}^{+}(\alpha)$, the linear form $\Phi(\mu)$ corresponds to integration against a $\mu$ stationary measure. Hence, (2.7) shows that the escape rate $\ell(\mu)$ is given by

$$
\ell(\mu)=\sum_{g \in \Gamma} \mu(g) \Phi(\mu)\left(\xi \mapsto c_{B}(g, \xi)\right) .
$$

We claim that the expression on the right defines an analytic function on $U$. Let us first show that the sum converges absolutely. We need to estimate the $C^{\beta}$ norm of $u_{g}: \xi \mapsto c_{B}(g, \xi)$. As $c_{B}(\cdot, \xi)$ is 1-Lipschitz and $c_{B}(e, \xi)=0$, we get $\left|c_{B}(g, \xi)\right| \leqslant|g|$, i.e., $u_{g}$ is bounded in sup norm by $|g|$. If $d\left(\xi, \xi^{\prime}\right) \leqslant e^{-|g|}$, we have $u_{g}(\xi)-u_{g}\left(\xi^{\prime}\right)=0$. On the other hand, if $d\left(\xi, \xi^{\prime}\right)>e^{-|g|}$, we use the sup norm bound by $|g|$ to write

$$
\left|u_{g}(\xi)-u_{g}\left(\xi^{\prime}\right)\right| / d\left(\xi, \xi^{\prime}\right)^{\beta} \leqslant 2|g| / e^{-\beta|g|} \leqslant C e^{\alpha|g|},
$$

where the last inequality follows as $\beta<\alpha$. Hence, $\left\|u_{g}\right\|_{C^{\beta}} \leqslant C e^{\alpha|g|}$. It follows that

$$
\sum_{g \in \Gamma}\left|\mu(g) \Phi(\mu)\left(u_{g}\right)\right| \leqslant \sum_{g \in \Gamma}|\mu(g)| C e^{\alpha|g|} \leqslant C\|\mu\|_{\alpha} .
$$

This shows that the right hand side of (3.1) is well defined. Moreover, each such sum, when restricted to a finite subset of $\Gamma$, is analytic. Since analytic functions are closed under uniform convergence, as we recalled in Paragraph 2.3, it follows that the sum over $\Gamma$ is also analytic. 


\section{ANALYTICITY OF THE ENTROPY AND THE EsCAPE RATE}

The rest of this section is devoted to the proof of Theorem 3.1.

To $\mu \in \mathcal{P}(\alpha)$ is associated a convolution operator on $\partial_{B} \Gamma$, and consequently an operator acting on continuous functions. It is given by

$$
L_{\mu} u(\xi)=\sum_{g} \mu(g) u(g \cdot \xi)
$$

The stationary measure $\Phi(\mu)$ of Theorem 3.1 will be constructed as an eigenfunction for the dual operator $L_{\mu}^{*}$ acting on $\left(C^{\beta}\right)^{*}$, for the eigenvalue 1 . Eigenfunctions corresponding to isolated eigenvalues which are simple depend analytically on the operator, hence we should understand the spectral properties of $L_{\mu}^{*}$ or, equivalently, of $L_{\mu}$.

The usual strategy, dating back to Le Page [LP82], is to prove the following contraction estimate:

$$
\sup _{\xi, \xi^{\prime} \in \partial_{B} \Gamma} \int\left(\frac{d\left(g \xi, g \xi^{\prime}\right)}{d\left(\xi, \xi^{\prime}\right)}\right)^{\beta} \mathrm{d} \mu^{* n}(g)<1,
$$

for some $n>0$ and some $\beta>0$. Such an estimate implies that $L_{\mu}$ has a simple eigenvalue at 1 and no other eigenvalue of modulus $\geqslant 1$, see for instance [Bjö10] or [BQ14]. Unfortunately, such an inequality can not hold in general in our context. For instance, in $\Gamma=F_{d} \times \mathbb{Z} / 2 \mathbb{Z}$, consider two horofunctions $\xi$ and $\xi^{\prime}$ coming from the two sheets $F_{d} \times\{0\}$ and $F_{d} \times\{1\}$. Then $g \xi$ and $g \xi^{\prime}$ are at distance 1 for any $g \in \Gamma$, since they differ on the element $e \times 1$, at distance 1 of the origin. Hence, (3.2) is always equal to 1 . In this example, one can nevertheless hope that $L_{\mu}$ has contraction properties due to another mechanism, maybe matching for instance $g \xi$ with $g^{\prime} \xi^{\prime}$ for another group element $g^{\prime}$.

In general, we will not obtain the contraction from an explicit contraction estimate such as (3.2), but rather from a less explicit spectral argument.

Proposition 3.3. For $\mu \in \mathcal{P}(\alpha)$, the operator $L_{\mu}$ acts continuously on $C^{0}$, and on $C^{\beta}$ when $\beta \leqslant \alpha$. Its operator norm is bounded by $\|\mu\|_{\alpha}$.

Proof. If $\mu$ has finite support, then $L_{\mu} u$ is the sum of the continuous functions $\xi \mapsto \mu(g) u(g \cdot \xi)$, bounded by $|\mu(g)|$. Hence, $L_{\mu}$ acts continuously on $C^{0}$ with norm at most $\sum|\mu(g)| \leqslant\|\mu\|_{\alpha}$. The general case follows by density.

Suppose now that two horofunctions $h_{\xi}$ and $h_{\xi^{\prime}}$ coincide on the ball $B(e, N)$. Then the horofunctions $h_{g \xi}$ and $h_{g \xi^{\prime}}$ coincide at least on the ball $B(e, N-|g|)$. Therefore,

$$
d\left(g \xi, g \xi^{\prime}\right) \leqslant e^{|g|} d\left(\xi, \xi^{\prime}\right) .
$$

Let $u \in C^{\beta}$. We have

$$
\begin{aligned}
\left|L_{\mu} u(\xi)-L_{\mu} u\left(\xi^{\prime}\right)\right| & \leqslant \sum\left|\mu(g)\left\|u(g \xi)-u\left(g \xi^{\prime}\right)\left|\leqslant \sum\right| \mu(g) \mid\right\| u \|_{C^{\beta}} d\left(g \xi, g \xi^{\prime}\right)^{\beta}\right. \\
& \leqslant \sum|\mu(g)|\|u\|_{C^{\beta}} e^{\beta|g|} d\left(\xi, \xi^{\prime}\right)^{\beta}=\|\mu\|_{\beta}\|u\|_{C^{\beta}} d\left(\xi, \xi^{\prime}\right)^{\beta} \\
& \leqslant\|\mu\|_{\alpha}\|u\|_{C^{\beta}} d\left(\xi, \xi^{\prime}\right)^{\beta} .
\end{aligned}
$$

This shows that $L_{\mu} u$ is again $\beta$-Hölder continuous, with Hölder constant at most $\|\mu\|_{\alpha}\|u\|_{C^{\beta}}$. 


\section{SÉBASTIEN GOUËZEL}

This computation does not give any contraction. To get contraction, we should show that $d\left(g \xi, g \xi^{\prime}\right)$ is smaller than $d\left(\xi, \xi^{\prime}\right)$ for most $g$. Starting from the fact that two horofunctions $h_{\xi}$ and $h_{\xi^{\prime}}$ coincide on a ball $B(e, N)$, we thus need to show that they coincide on a bigger ball centered at $g^{-1}$. This is essentially the content of the following lemma.

Lemma 3.4. Let $h_{1}$ and $h_{2}$ be two horofunctions, which coincide on a ball $B(e, N)$. Let $x \in B(e, N-10 \delta)$. Then $h_{1}$ and $h_{2}$ coincide on the ball $B\left(x, N+h_{1}(x)-10 \delta\right)$.

This lemma is trivial for $x=e$, or more generally when $h_{1}(x)=-d(e, x)$ : in this case, the ball $B\left(x, N+h_{1}(x)-10 \delta\right)$ is included in $B(e, N)$, where we already know that $h_{1}$ and $h_{2}$ coincide. On the other hand, it gives new and useful information for instance when $h_{1}(x)$ is positive. This lemma follows easily from the arguments in [CP01], as we explain now. The idea is that geodesics from a point $y \in B\left(x, N+h_{1}(x)-10 \delta\right)$ to the point at infinity directed by $h_{1}$ have to enter $B(e, N)$, hence the value of $h_{1}$ on $y$ is determined by its values on $B(e, N)$. The reader should keep this geometric picture in mind, although the rigorous formalization in terms of Gromov products is rather tedious.

Proof. We will use the following easy inequality on Gromov products. For any $u, v$ and $w$, one has $d(w, v) \geqslant d(w, u)-d(u, v)$. Hence

$$
(u \mid v)_{w}=(d(w, u)+d(w, v)-d(u, v)) / 2 \geqslant d(w, u)-d(u, v) .
$$

Take $x \in B(e, N-10 \delta)$. As $h_{1}$ is a horofunction, it is a pointwise limit of normalized distance functions. Hence, we may take $z$ with $d(e, z)>2 N$ such that $h_{1}(y)=h_{z}(y)$ for all $y \in B(e, 2 N)$, where $h_{z}(y)=d(z, y)-d(z, e)$. Write $d(e, z)=M+N$ with $M>N$. Then

$$
(e \mid x)_{z} \geqslant d(z, e)-d(e, x) \geqslant M+N-(N-10 \delta)=M+10 \delta .
$$

Consider $y \in B\left(x, N+h_{1}(x)-10 \delta\right)$. As $d(z, x)=d(z, e)+h_{z}(x)=M+N+h_{1}(x)$, we obtain

$$
(x \mid y)_{z} \geqslant d(z, x)-d(y, x) \geqslant M+N+h_{1}(x)-\left(N+h_{1}(x)-10 \delta\right)=M+10 \delta .
$$

The inequality (2.1) characterizing hyperbolic spaces entails

$$
(e \mid y)_{z} \geqslant M+9 \delta .
$$

Consider $a$ and $b$ the points on geodesics from $z$ to $e$ and from $z$ to $y$, at distance $M+9 \delta$ of $z$. They belong to $\Gamma$ since $\delta \in \mathbb{N}$ by assumption. Moreover, $d(e, a)=N-9 \delta$. We have $(e \mid a)_{z}=M+9 \delta$ and $(y \mid b)_{z}=M+9 \delta$. Combining these two inequalities with hyperbolicity, and using (3.4), we get

$$
(a \mid b)_{z} \geqslant M+7 \delta \text {. }
$$

Hence,

$$
d(a, b)=d(a, z)+d(b, z)-2(a \mid b)_{z} \leqslant 2(M+9 \delta)-2(M+7 \delta)=4 \delta .
$$

In particular, $d(b, e) \leqslant d(a, e)+4 \delta \leqslant N-5 \delta$. This implies that $b \in B(e, N)$, so that $h_{1}(b)=h_{2}(b)$.

As $b$ is on a geodesic segment from $z$ to $y$, we have

$$
h_{1}(y)=h_{z}(y)=h_{z}(b)+d(b, y)=h_{1}(b)+d(b, y) .
$$




\section{ANALYTICITY OF THE ENTROPY AND THE ESCAPE RATE}

Moreover, as $h_{2}$ is 1 -Lipschitz, $h_{2}(y) \leqslant h_{2}(b)+d(b, y)$. This shows that $h_{2}(y) \leqslant h_{1}(y)$.

We have proved that $h_{2} \leqslant h_{1}$ on $B\left(x, N+h_{1}(x)-10 \delta\right)$. Since everything is symmetric, the reverse inequality and the equality follow.

A weak form of contraction of the convolution operator (called a Doeblin-Fortet inequality) follows using the standard trick of Le Page [LP82], as explained for instance in [BQ14, Lemma 12.6].

Lemma 3.5. Let $\mu \in \mathcal{P}_{1}^{+}(\alpha)$. There exist $n>0, \beta \leqslant \alpha / 2, C>0$ and $\rho<1$ such that, for any $u \in C^{\beta}$,

$$
\left\|L_{\mu}^{n} u\right\|_{C^{\beta}} \leqslant \rho\|u\|_{C^{\beta}}+C\|u\|_{C^{0}} .
$$

Proof. Let $\beta \leqslant \alpha / 2$. Fix $n \in \mathbb{N}$, and $N>0$. As the sup norm control $\left\|L \mu^{n} u\right\|_{C^{0}} \leqslant\|u\|_{C^{0}}$ is trivial, we just have to estimate the $\beta$-Hölder constant of $L_{\mu}^{n} u$. Given $\xi$ and $\xi^{\prime}$, we should bound $\left|L_{\mu}^{n} u(\xi)-L_{\mu}^{n} u\left(\xi^{\prime}\right)\right|$. If $d\left(\xi, \xi^{\prime}\right)>e^{-N}$, we simply write

$$
\left|L_{\mu}^{n} u(\xi)-L_{\mu}^{n} u\left(\xi^{\prime}\right)\right| \leqslant 2\left\|L_{\mu}^{n} u\right\|_{C^{0}} \leqslant 2\|u\|_{C^{0}} \leqslant d\left(\xi, \xi^{\prime}\right)^{\beta} \cdot 2 \cdot e^{\beta N}\|u\|_{C^{0}} .
$$

This is compatible with the inequality we seek, for a large $C \geqslant 2 e^{\beta N}$.

Assume now that $d\left(\xi, \xi^{\prime}\right)=e^{-M} \leqslant e^{-N}$, i.e., the two horofunctions $h_{\xi}$ and $h_{\xi^{\prime}}$ coincide on the ball $B(e, M)$ with $M \geqslant N$. When $|g| \leqslant N-10 \delta$, Lemma 3.4 implies that $h_{\xi}$ and $h_{\xi^{\prime}}$ coincide on the ball $B\left(g^{-1}, M+h_{\xi}\left(g^{-1}\right)-10 \delta\right)$. We recall that $g \cdot \xi$ satisfies $h_{g \xi}(x)=h_{\xi}\left(g^{-1} x\right)-h_{\xi}\left(g^{-1}\right)$. Hence, $h_{g \xi}$ and $h_{g \xi^{\prime}}$ coincide on the ball $B\left(e, M+h_{\xi}\left(g^{-1}\right)-10 \delta\right)$. This yields $d\left(g \xi, g \xi^{\prime}\right) \leqslant e^{10 \delta-h_{\xi}\left(g^{-1}\right)} d\left(\xi, \xi^{\prime}\right)$. If $|g|>N-10 \delta$, we simply use the trivial inequality $d\left(g \xi, g \xi^{\prime}\right) \leqslant e^{|g|} d\left(\xi, \xi^{\prime}\right)$ proved in (3.3) instead.

We obtain

$$
\begin{aligned}
\left|L_{\mu}^{n} u(\xi)-L_{\mu}^{n} u\left(\xi^{\prime}\right)\right| \leqslant & \sum_{g} \mu^{* n}(g)\left|u(g \xi)-u\left(g \xi^{\prime}\right)\right| \leqslant \sum_{g} \mu^{* n}(g)\|u\|_{C^{\beta}} d\left(g \xi, g \xi^{\prime}\right)^{\beta} \\
& \leqslant\|u\|_{C^{\beta}} d\left(\xi, \xi^{\prime}\right)^{\beta}\left(\sum_{|g| \leqslant N-10 \delta} \mu^{* n}(g) e^{\beta\left(10 \delta-h_{\xi}\left(g^{-1}\right)\right)}+\sum_{|g|>N-10 \delta} \mu^{* n}(g) e^{\beta|g|}\right) .
\end{aligned}
$$

If the term between parenthesis on the last line is bounded by $\rho<1$, uniformly in $\xi \in \partial_{B} \Gamma$, then we get $\left|L_{\mu}^{n} u(\xi)-L_{\mu}^{n} u\left(\xi^{\prime}\right)\right| \leqslant \rho\|u\|_{C^{\beta}} d\left(\xi, \xi^{\prime}\right)^{\beta}$, which is the desired bound for the pair $\xi, \xi^{\prime}$.

Hence, to conclude, it suffices to obtain such a bound by $\rho<1$ as above. It is even sufficient to obtain it for $N=\infty$, since the same bound (with a slightly larger $\rho^{\prime} \in(\rho, 1)$ ) then follows for any large enough finite $N$. Finally, it suffices to find $\beta \leqslant \alpha / 2$ and $n$ such that, uniformly in $\xi \in \partial_{B} \Gamma$,

$$
\sum_{g} \mu^{* n}(g) e^{\beta\left(10 \delta-h_{\xi}\left(g^{-1}\right)\right)} \leqslant \rho<1 .
$$

We use the inequality $e^{t} \leqslant 1+t+t^{2} e^{|t|}$. Moreover, there exists $C$ such that $t^{2} \leqslant C e^{\alpha|t| / 2}$ for all real $t$. Hence, the sum in the above equation is bounded by

$$
\begin{aligned}
\sum_{g} \mu^{* n}(g)(1+\beta(10 \delta & \left.\left.-h_{\xi}\left(g^{-1}\right)\right)+\beta^{2}\left(10 \delta-h_{\xi}\left(g^{-1}\right)\right)^{2} e^{\beta(10 \delta+|g|)}\right) \\
& \leqslant 1-\beta\left(\sum_{g} \mu^{* n}(g)\left(h_{\xi}\left(g^{-1}\right)-10 \delta\right)\right)+\beta^{2} C\left(\sum_{g} \mu^{* n}(g) e^{(\beta+\alpha / 2)(10 \delta+|g|)}\right) .
\end{aligned}
$$


When $\beta \leqslant \alpha / 2$, the last sum is finite and bounded by $e^{10 \alpha \delta}\left\|\mu^{* n}\right\|_{\alpha}$. Hence, when $\beta$ tends to 0 , the last term is $O\left(\beta^{2}\right)$, and is negligible with respect to the first one, of order $\beta$. If the first term is strictly negative, (3.6) follows for small enough $\beta$. Therefore, it suffices to show that

$$
\sum_{g} \mu^{* n}(g)\left(h_{\xi}\left(g^{-1}\right)-10 \delta\right) \geqslant K>0,
$$

uniformly in $\xi \in \partial_{B} \Gamma$. The term on the left of this equation looks closely like the escape rate of the random walk, which is strictly positive. The difficulty is that we want a pointwise uniform inequality, not an averaged version.

This kind of situation has already been encountered several times in the literature, hence efficient tools are available. We recall for instance Theorem 2.9 in [BQ14]. Let $\Gamma$ be a countable group acting continuously on a compact space $X$, let $\mu$ be a probability measure on $\Gamma$, and let $c: \Gamma \times X \rightarrow \mathbb{R}$ be a continuous cocycle, i.e., a continuous function satisfying $c\left(g_{1} g_{2}, x\right)=c\left(g_{1}, g_{2} x\right)+c\left(g_{2}, x\right)$. Assume that $\sum_{g \in \Gamma} \mu(g) \sup _{x \in X}|c(g, x)|<\infty$. Assume also that there exists $\ell \in \mathbb{R}$ such that, for any $\mu$-stationary probability measure $v$ on $X, \int_{\Gamma \times X} c(g, x) \mathrm{d} \mu(g) \mathrm{d} v(x)=\ell$. Then, as $n \rightarrow \infty$,

$$
\frac{1}{n} \sum_{g \in \Gamma} \mu^{* n}(g) c(g, x) \rightarrow \ell
$$

uniformly in $x \in X$.

We will apply this statement to the Busemann cocycle on $X=\partial_{B} \Gamma$, given by $c_{B}(g, \xi)=h_{\xi}\left(g^{-1}\right)$. In this case, for any stationary measure $v$ on $\partial_{B} \Gamma,(2.7)$ states that

$$
\int_{X} c_{B}(g, \xi) \mathrm{d} \mu(g) \mathrm{d} v(\xi)=\ell .
$$

Hence, we deduce from [BQ14, Theorem 2.9] that

$$
\frac{1}{n} \sum_{g \in \Gamma} \mu^{* n}(g) c_{B}(g, \xi) \rightarrow \ell, \text { uniformly in } \xi \in \partial_{B} \Gamma .
$$

As $\mu$ is admissible and $\Gamma$ is non-amenable, $\ell>0$. Hence, if $n$ is large enough, we have for all $\xi \in \partial_{B} \Gamma$ the inequality

$$
\sum_{g} \mu^{* n}(g) h_{\xi}\left(g^{-1}\right) \geqslant n \ell / 2 .
$$

This implies (3.7) if $n$ is large enough so that $n \ell / 2>10 \delta$.

The group $\Gamma$ acts continuously on the compact set $\partial_{B} \Gamma$. By Zorn's lemma, there exists a nonempty compact invariant subset of $\partial_{B} \Gamma$ which is minimal among such sets. It is simply called a minimal subset of $\partial_{B} \Gamma$. An essential feature of such a set $\partial_{B}^{\prime} \Gamma$ is that, for any $x \in \partial_{B}^{\prime} \Gamma$, then $\Gamma x$ is dense in $\partial_{B}^{\prime} \Gamma$. Indeed, otherwise, the closure of $\Gamma x$ would be a nonempty compact invariant strict subset of $\partial_{B}^{\prime} \Gamma$, contradicting its minimality.

Proposition 3.6. Let $\mu \in \mathcal{P}_{1}^{+}(\alpha)$. Consider a nonempty compact subset $\partial_{B}^{\prime} \Gamma \subseteq \partial_{B} \Gamma$ which is minimal for the $\Gamma$-action. Then, for small enough $\beta$, the operator $L_{\mu}$ acting on $C^{\beta}\left(\partial_{B}^{\prime} \Gamma\right)$ has a simple eigenvalue at 1 , finitely many eigenvalues of modulus 1 (they are simple and do not have nontrivial Jordan blocks) and the rest of its spectrum is contained in a disk $D(0, r)$ for some $r<1$. 
Proof. In the proof, we let $L_{\mu}$ act on $C^{\beta}\left(\partial_{B}^{\prime} \Gamma\right)$. On this space, the estimates of Lemma 3.5 readily follow from the corresponding ones on $C^{\beta}\left(\partial_{B} \Gamma\right)$. Let $n$ and $\beta$ be given by Lemma 3.5. In the inequality (3.5), the term $\rho\|u\|_{C^{\beta}}$ heuristically corresponds to a part of $L_{\mu}$ with spectral radius at most $\rho^{1 / n}$, while the term $C\|u\|_{C^{0}}$ would come from a compact part (as the inclusion of $C^{\beta}$ in $C^{0}$ is compact), which should only add discrete eigenvalues. This intuition is made precise by a theorem of Hennion [Hen93]: It entails that (3.5) implies that the spectrum of $L_{\mu}$ in $\left\{z \in \mathbb{C}:|z|>\rho^{1 / n}\right\}$ is made of isolated eigenvalues of finite multiplicity.

In particular, by discreteness, there are finitely many eigenvalues of modulus $\geqslant 1$, and the rest of the spectrum is contained in a disk $D(0, r)$ for some $r<1$. As the iterates of $L_{\mu}$ on $C^{0}$ are uniformly bounded, there is no eigenvalue with modulus $>1$. Moreover, the eigenvalues of modulus 1 have no Jordan block.

Let $u$ be a nonzero eigenfunction of $L_{\mu}$ for an eigenvalue $\rho$ of modulus 1 . Then $v=|u|$ satisfies

$$
v=|u|=\left|L_{\mu}^{k} u\right| \leqslant L_{\mu}^{k}|u|=L_{\mu}^{k} v .
$$

Consider $\xi \in \partial_{B}^{\prime} \Gamma$ such that $v(\xi)$ is maximal. Then the previous inequality implies that $v(g \xi)=v(\xi)$ for all $g$ in the semigroup generated by the support of $\mu$. By admissibility, this is true for all $g \in \Gamma$. The orbit of $\xi$ is dense in $\partial_{B}^{\prime} \Gamma$ by minimality. Thus, $v$ is constant.

The equality in (3.8) also implies that all the complex numbers $u(g \xi)$ for $g \in \operatorname{supp}\left(\mu^{* k}\right)$ have the same phase. Hence, $u$ is constant on $\left(\operatorname{supp} \mu^{* k}\right) \xi$.

We claim that

$$
\text { there exists } N>0 \text { with } \mu^{* N}(e)>0 .
$$

Indeed, fix $x \neq e$. Then, by admissibility, there is $\ell_{1}>0$ such that $\mu^{* \ell_{1}}(x)>0$, and $\ell_{2}>0$ such that $\mu^{* \ell_{2}}\left(x^{-1}\right)>0$. Then $\mu^{*\left(\ell_{1}+\ell_{2}\right)}(e)>0$.

Fix such an $N$. Then, for any $j$, the sequence $\left(\operatorname{supp} \mu^{*(k N+j)}\right)(\xi)$ increases with $k$, towards a limiting set $A_{j} \subseteq \partial_{B}^{\prime} \Gamma$. The function $u$ is constant on each set $\overline{A_{j}}$. Moreover, for $j \in \mathbb{Z} / N \mathbb{Z}$, one has $(\operatorname{supp} \mu) \cdot A_{j}=A_{j+1}$. As $L_{\mu} u=\rho u$, it follows that $\rho u_{\mid \overline{A_{j}}}=u_{\mid \overline{A_{j+1}}}$. Finally, one gets $u(x)=\rho^{j} u(\xi)$ for $x \in \overline{A_{j}}$. As $\bigcup_{j} \overline{A_{j}}=\partial_{B}^{\prime} \Gamma$ by minimality, this shows that $u$ is determined by its value at $\xi$, and therefore that the $\rho$-eigenspace is at most 1 -dimensional.

For $\rho=1$, the eigenspace is exactly 1-dimensional, as it contains the constant functions.

Example. On $\Gamma=F_{2} \times \mathbb{Z} / 2 \mathbb{Z}$, consider the uniform measure $\mu$ on $(e, 1),(a, 1),\left(a^{-1}, 1\right),(b, 1)$ and $\left(b^{-1}, 1\right)$, where $a$ and $b$ are the canonical generators of $F_{2}$. It is admissible. Let $v$ denote its (unique) stationary measure on the Gromov boundary $\partial \Gamma \simeq \partial F_{2}$. The Busemann boundary $\partial_{B} \Gamma$ is canonically isomorphic to $\partial F_{2} \times \mathbb{Z} / 2 \mathbb{Z}$, and the $\Gamma$ action on $\partial_{B} \Gamma$ is minimal. Write $v_{0}$ and $v_{1}$ for $v \otimes \delta_{0}$ and $v \otimes \delta_{1}$. As the measure $\mu$ exchanges the sheets $F_{2} \times\{0\}$ and $F_{2} \times\{1\}$, the operator $\left(L_{\mu}\right)^{*}$ maps $v_{0}$ to $v_{1}$ and $v_{1}$ to $v_{0}$. Dually, denoting by $u_{i}$ the characteristic function of $\partial F_{2} \times\{i\}$ for $i=0,1$, the operator $L_{\mu}$ maps $u_{0}$ to $u_{1}$ and $u_{1}$ to $u_{0}$. The constant function $u=u_{0}+u_{1}=2$ is invariant under $L_{\mu}$. Moreover, this operator also has a simple eigenvalue at -1 , for the eigenfunction $u_{0}-u_{1}$. There is no other eigenvalue of modulus 1. Dually, the measure $v_{0}+v_{1}$ is an eigenmeasure of $\left(L_{\mu}\right)^{*}$ for the eigenvalue 1 and $v_{0}-v_{1}$ is an eigenmeasure for the eigenvalue -1 .

Proof of Theorem 3.1. It suffices to prove the theorem for small enough $\beta$, as $\left(C^{\beta}\right)^{*} \subseteq\left(C^{\beta^{\prime}}\right)^{*}$ when $\beta \leqslant \beta^{\prime}$. We will use basic results of spectral theory in Banach spaces, as explained for instance in [Kat66]. 


\section{SÉBASTIEN GOUËZEL}

Take $\mu_{0} \in \mathcal{P}_{1}^{+}(\alpha)$. Proposition 3.6 applies to $\mu_{0}$. Hence, for small enough $\beta$, one can decompose the space $C^{\beta}\left(\partial_{B}^{\prime} \Gamma\right)$ as a direct sum of finite-dimensional eigenspaces $E_{\rho}$ associated to eigenvalues $\rho$ with modulus 1 , and an infinite-dimensional subspace $E_{<1}$ on which $L_{\mu_{0}}^{n}$ tends exponentially fast to 0 . Let $\Pi_{\mu_{0}}$ be the eigenprojection for the eigenvalue 1, i.e., the projection on $E_{1}$ with kernel $E_{<1} \oplus \bigoplus_{\rho \neq 1} E_{\rho}$. Then we claim that, for any $u \in C^{\beta}\left(\partial_{B}^{\prime} \Gamma\right)$,

$$
\frac{1}{k} \sum_{i=0}^{k-1} L_{\mu_{0}}^{i} u \rightarrow \Pi_{\mu_{0}} u
$$

Indeed, this is clear on each $E_{\rho}$ and on $E$ separately, and the general result follows. By [Kat66], the projection $\Pi_{\mu_{0}}$ is also given by the formula

$$
\Pi_{\mu_{0}} u=\frac{1}{2 \mathbf{i} \pi} \int_{\mathcal{C}}\left(z I-L_{\mu_{0}}\right)^{-1} \mathrm{~d} z
$$

where $\mathcal{C}$ is any small enough circle around 1.

The map $\mu \mapsto L_{\mu}$ is linear and continuous by Proposition 3.3 (and therefore analytic). For $\mu$ close enough to $\mu_{0}$, the operator $L_{\mu}$ is close to $L_{\mu_{0}}$. Hence, the spectral description given by Proposition 3.6 for $L_{\mu_{0}}$ persists for $L_{\mu}$ by spectral continuity results for isolated simple eigenvalues, see [Kat66]: the operator $L_{\mu}$ has a unique eigenvalue close to 1 , it is simple, and the corresponding spectral projection $\Pi_{\mu}$ is given by the formula (3.11) (with $\mu_{0}$ replaced by $\mu$ ). Moreover, $\mu \mapsto \Pi_{\mu}$ is analytic. When $\mu \in \mathcal{P}_{1}^{+}(\alpha)$, the operator $L_{\mu}$ is a convolution operator with a probability measure, hence $L_{\mu} 1=1$. In particular, the corresponding eigenvalue is 1 .

Let $\xi_{0} \in \partial_{B}^{\prime} \Gamma$. We define a linear form $\Phi(\mu)$ on $C^{\beta}\left(\partial_{B}^{\prime} \Gamma\right)$, for $\mu$ close to $\mu_{0}$, by $\Phi(\mu)(u)=\left(\Pi_{\mu} u\right)\left(\xi_{0}\right)$. Then $\mu \mapsto \Phi(\mu)$ is analytic from $U$ to $\left(C^{\beta}\left(\partial_{B}^{\prime} \Gamma\right)\right)^{*}$. As Hölder functions on $\partial_{B} \Gamma$ restrict to Hölder functions on $\partial_{B}^{\prime} \Gamma, \Phi(\mu)$ can be considered as a linear form on $C^{\beta}\left(\partial_{B} \Gamma\right)$. Equivalently, we implicitly compose $\Phi(\mu)$ with the continuous inclusion $\left(C^{\beta}\left(\partial_{B}^{\prime} \Gamma\right)\right)^{*} \subseteq\left(C^{\beta}\left(\partial_{B} \Gamma\right)\right)^{*}$. Hence, $\Phi(\mu) \in\left(C^{\beta}\left(\partial_{B} \Gamma\right)\right)^{*}$.

It remains to show that the linear form $\Phi(\mu)$ is the integration against a stationary probability measure on $\partial_{B}^{\prime} \Gamma$ when $\mu \in \mathcal{P}_{1}^{+}(\alpha)$. In this case, $\Phi(\mu)(u)$ is again given by the formula (3.10) (with $\mu_{0}$ replaced by $\mu$ ), as the above discussion also applies to $\mu$. For any nonnegative Hölder function $u$, one gets $0 \leqslant \Phi(\mu)(u) \leqslant\|u\|_{C^{0}}$. By Stone-Weierstrass theorem, Hölder functions are dense in $C^{0}$. We deduce that $\Phi(\mu)$ extends to a positive linear form on continuous functions, i.e., a positive measure on $\partial_{B}^{\prime} \Gamma$. As $\Phi(\mu) 1=1$, it is a probability measure. Finally, as $\Phi(\mu)\left(L_{\mu} u\right)=\Phi(\mu)(u)$, it is stationary.

\section{Analyticity of the entropy}

Let $\Gamma$ be a nonelementary hyperbolic group. We fix once and for all a finite subset $F$ of $\Gamma$. We denote by $\mathcal{P}(F)$ the set of functions $\mu: F \rightarrow \mathbb{C}$, and by $\mathcal{P}_{1}^{+}(F)$ its subset made of admissible probability measures. We also fix a reference measure $\mu_{0} \in \mathcal{P}_{1}^{+}(F)$. Note that the support of $\mu_{0}$ may be a proper subset of $F$. In this section, we prove the entropy part of Theorem 1.1:

Theorem 4.1. The map $\mu \mapsto h(\mu)$, associating to $\mu \in \mathcal{P}_{1}^{+}(F)$ its entropy, extends to an analytic map on a neighborhood of $\mu_{0}$ in $\mathcal{P}(F)$.

The idea is to start from (2.10), and to show that the lift $K_{\mu}^{B}(x, \xi)$ of the Martin kernel to the Busemann boundary depends analytically on $\mu$. 


\subsection{Strong Ancona inequalities}

A fundamental tool to study the Green function and the Martin kernel in hyperbolic groups is an inequality due to Ancona, showing that the Green function is essentially multiplicative along geodesics. In other words, typical trajectories of the random walk tend to follow geodesics. We will use repeatedly a quantitative version of such inequalities, that we describe in this paragraph. As it should hold uniformly on a neighborhood of $\mu_{0}$, we first describe such a convenient neighborhood.

As $\Gamma$ is non-amenable, the spectral radius of the convolution operator by $\mu_{0}$ on $\ell^{2}(\Gamma)$ is $<1$. We fix $\varepsilon_{0}>0$ small enough so that the measure $\bar{\mu}=\mu_{0}+\varepsilon_{0} \sum_{g \in F} \delta_{g}$ also has a spectral radius $<1$.

We denote by $\bar{U} \subseteq \mathcal{P}(F)$ the set of functions supported on $F$ with $\left|\mu(g)-\mu_{0}(g)\right| \leqslant \varepsilon_{0}$ for all $g \in F$, and by $U$ its interior. We also write $\bar{U}^{+}$for the nonnegative elements of $\bar{U}$, and $\bar{U}_{1}^{+}$for the admissible probability measures in $\bar{U}$. As all functions in $\bar{U}$ are dominated by $\bar{\mu}$, they all have a spectral radius $<1$, uniformly. Moreover, the Green function (defined in (2.8)) of $\mu \in \bar{U}^{+}$satisfies

$$
G_{\mu}(x, y) \leqslant G_{\bar{\mu}}(x, y)
$$

for all $x, y \in \Gamma$.

If $\varepsilon_{0}$ is small enough, all measures $\mu \in \bar{U}^{+}$satisfy $\mu(g) \geqslant \mu_{0}(g) / 2$ for all $g \in \operatorname{supp} \mu_{0}$. As $\mu_{0}$ is admissible, it follows that $G_{\mu}(x, y) \geqslant e^{-C d(x, y)}$ for all $x, y \in \Gamma$, uniformly in $\mu$. As trajectories from $x$ to $y$ and trajectories from $y$ to $z$ can be concatenated to form trajectories from $x$ to $z$, we deduce the following Harnack inequalities, uniformly in $x, y, z \in \Gamma$ and $\mu \in \bar{U}^{+}$:

$$
e^{-C d(x, y)} \leqslant \frac{G_{\mu}(x, z)}{G_{\mu}(y, z)} \leqslant e^{C d(x, y)}, \quad e^{-C d(y, z)} \leqslant \frac{G_{\mu}(x, y)}{G_{\mu}(x, z)} \leqslant e^{C d(y, z)} .
$$

For $\mu \in \bar{U}^{+}$, we say that a function $u: \Gamma \rightarrow \mathbb{R}$ is $\mu$-harmonic in a domain $A$ of $\Gamma$ if, for all $x \in A$,

$$
u(x)=\sum_{g} \mu(g) u(x g)
$$

For instance, the Green function $x \mapsto G_{\mu}(x, y)$ is $\mu$-harmonic on $\Gamma \backslash\{y\}$.

Consider a geodesic $\gamma$ in $\Gamma$, with length $D \geqslant 0$, from $x_{0}=\gamma(0)$ to $y_{0}=\gamma(D)$. We define a domain of points close to the beginning of $\gamma$ as $I^{-}(\gamma)=\left\{x \in \Gamma:\left(x_{0} \mid x\right)_{y_{0}} \geqslant D-10 \delta\right\}$. In the same way, let $I^{+}(\gamma)=\left\{y \in \Gamma:\left(y_{0} \mid y\right)_{x_{0}} \geqslant D-10 \delta\right\}$.

The quantitative Ancona inequalities we will use are the following:

Proposition 4.2. There exist $C>0$ and $D_{0}$ such that, for all $\mu \in \bar{U}^{+}$, the following holds. Let $\gamma$ be a geodesic segment in $\Gamma$, with length $D \geqslant D_{0}$. Let $u$ and $v$ be two nonnegative functions on $\Gamma$ which satisfy the following outside of $I^{+}(\gamma)$ : they are strictly positive, $\mu$-harmonic, and bounded by a finite linear combination of functions $G_{\mu}(\cdot, y)$ where $y \in I^{+}(\gamma)$. Then, for all $x, x^{\prime} \in I^{-}(\gamma)$

$$
\left|\frac{u(x) / u\left(x^{\prime}\right)}{v(x) / v\left(x^{\prime}\right)}-1\right| \leqslant C e^{-C^{-1} D}
$$


Proof. This follows from the arguments in [GL13b]. Indeed, the conclusion of Lemma 4.4 there is a consequence of the fact that the spectral radius is bounded away from 1 on $\bar{U}^{+}$. Then all the arguments up to the end of Theorem 4.6 apply verbatim, even for general $\mu$-harmonic functions. Note that, while it is assumed in the statements of [GL13b] that the measure is symmetric, this is only used in the proof of Lemma 4.4 there (which is trivial in our context, even without symmetry).

One important tool in this proof is the Ancona inequality (Theorem 4.1 in [GL13b]), saying that the Green function is multiplicative up to a constant along geodesics. We will use the following version:

Lemma 4.3. For any $K>0$, there exists $C>0$ with the following property. Let $\mu \in \bar{U}^{+}$. Consider three points $x, y, z$ in $\Gamma$ such that $z$ is within distance $K$ of a geodesic segment between $x$ and $y$. Then

$$
C^{-1} G_{\mu}(x, z) G_{\mu}(z, y) \leqslant G_{\mu}(x, y) \leqslant C G_{\mu}(x, z) G_{\mu}(z, y) .
$$

To illustrate the use of Proposition 4.2, let us explain how, conversely, it implies Ancona inequalities. We stress that this is not the logical order, as the proof of Proposition 4.2 uses the lemma.

Proof. By Harnack inequalities, it suffices to prove the lemma when $K=0$, i.e., $z$ belongs to a geodesic between $x$ and $y$.

If $z$ is $D_{0}$-close to $x$ or $y$, then Ancona inequalities are trivial thanks to Harnack inequalities (4.1). Otherwise, along a geodesic from $x$ to $y$ containing $z$, consider the point $x_{0}$ between $x$ and $z$ at distance $D=D_{0}$ of $z$, and similarly the point $y_{0}$ between $z$ and $y$ with $d\left(z, y_{0}\right)=D_{0}$. Let $\gamma$ be the restriction of the geodesic to $\left[x_{0} y_{0}\right]$. Then $x, x_{0} \in I^{-}(\gamma)$ and $y, y_{0} \in I^{+}(\gamma)$. The functions $u=G_{\mu}(\cdot, y)$ and $v=G_{\mu}\left(\cdot, y_{0}\right)$ satisfy the assumptions of the proposition. We deduce

$$
\left|\frac{G_{\mu}(x, y) / G_{\mu}\left(x_{0}, y\right)}{G_{\mu}\left(x, y_{0}\right) / G_{\mu}\left(x_{0}, y_{0}\right)}-1\right| \leqslant C e^{-C^{-1} D_{0}} .
$$

In particular, the fraction is bounded. As $x_{0}$ and $y_{0}$ are within bounded distance of $z$, this fraction coincides, up to a multiplicative constant, with

$$
\frac{G_{\mu}(x, y) / G_{\mu}(z, y)}{G_{\mu}(x, z) / G_{\mu}(z, z)} .
$$

Its boundedness shows that $G_{\mu}(x, y) \leqslant C G_{\mu}(x, z) G_{\mu}(z, y)$. Conversely, the other inequality $G_{\mu}(x, y) \geqslant$ $C^{-1} G_{\mu}(x, z) G_{\mu}(z, y)$ is trivial since trajectories from $x$ to $z$ and from $z$ to $y$ can be concatenated to form trajectories from $x$ to $y$.

Remark 4.4. Proposition 4.2 applies to the Martin kernel $x \mapsto K_{\mu}(x, \xi)$ when $\xi \notin \overline{\Gamma \backslash I^{+}(\gamma)}$. It is $\mu$ harmonic and positive everywhere, what remains to be checked is that it is bounded by $C G_{\mu}\left(x, y_{0}\right)$. Let $y_{n} \in I^{+}(\gamma)$ tend to $\xi$. Then a geodesic from $x$ to $y_{n}$ passes within bounded distance of $y_{0}$, uniformly in $n$ and in $x$ in $\Gamma \backslash I^{+}(\gamma)$. Thus, Ancona inequalities give

$$
G_{\mu}\left(x, y_{n}\right) \leqslant C G_{\mu}\left(x, y_{0}\right) G_{\mu}\left(y_{0}, y_{n}\right) .
$$

By Harnack inequalities, $G_{\mu}\left(y_{0}, y_{n}\right) \leqslant C G_{\mu}\left(e, y_{n}\right)$. Hence,

$$
G_{\mu}\left(x, y_{n}\right) / G_{\mu}\left(e, y_{n}\right) \leqslant C G_{\mu}\left(x, y_{0}\right) .
$$

Letting $n$ tend to infinity, we obtain $K_{\mu}(x, \xi) \leqslant C G_{\mu}\left(x, y_{0}\right)$ as desired. 


\section{ANALYTICITY OF THE ENTROPY AND THE EsCAPE RATE}

When $A$ is a subset of $\Gamma$ and $\mu \in \bar{U}^{+}$, we define the relative Green function $G_{\mu}(x, y ; A)$ as the sum of $\mu$-probabilities of paths from $x$ to $y$ that stay in $A$ except possibly at the first and last step, i.e.,

$$
G_{\mu}(x, y ; A)=\sum_{n \geqslant 0} \sum_{\substack{x_{0}=x, x_{1}, \ldots, x_{n}=y \\ x_{1}, \ldots, x_{n-1} \in A}} \mu\left(x_{0}^{-1} x_{1}\right) \cdots \mu\left(x_{n-1}^{-1} x_{n}\right) .
$$

The function $x \mapsto G_{\mu}(x, y ; A)$ is harmonic on the set of points $x$ that are different from $y$ and can not jump outside of $A$ in one step. It is bounded by $G_{\mu}(x, y)$. Hence, Proposition 4.2 applies to relative Green functions on suitable domains. One has $G_{\mu}(x, y ; \Gamma)=G_{\mu}(x, y)$.

\subsection{The Martin kernel as a fixed point}

To prove that the entropy depends analytically on the measure, using (2.10), we should show that the lifted Martin kernel $K_{\mu}^{B}$ depends analytically on $\mu$. As we explained in the introduction, our strategy is to exhibit $K_{\mu}^{B}$ as the fixed point of a suitable operator, and conclude using the implicit function theorem.

We will now introduce this operator, first formally. Let $N>0$ be large enough, only depending on the subset $F$ of $\Gamma$ in which the measures we consider are supported. To $\xi \in \partial_{B} \Gamma$, we associate a point $a(\xi) \in \Gamma$ with $|a(\xi)|=N$ which, heuristically, points in the direction of $\xi$. We require that $h_{\xi}(a(\xi))=-N$ and that $a(\xi)$ only depends on the restriction of $h_{\xi}$ to $B(e, N)$. We also associate to $\xi$ a set of points in $\Gamma$ denoted by $\Lambda(\xi)=\Lambda_{0}(\xi)$, which only depends on the restriction of $h_{\xi}$ to $B(e, N)$ and contains the points "far away from $\xi$ ". More precisely, let

$$
\Lambda(\xi)=\left\{g \in \Gamma:(a(\xi) \mid g)_{e} \leqslant N / 4\right\} .
$$

If $N$ is large enough, $F$ and $F^{-1}$ are included in $\Lambda(\xi)$ for every $\xi$. We will also need a slightly larger set

$$
\Lambda^{\prime}(\xi)=\left\{g \in \Gamma:(a(\xi) \mid g)_{e} \leqslant N / 2\right\} .
$$

Given $\xi \in \partial_{B} \Gamma$, there is now a canonical way to go towards $\xi$ at infinity, starting from the identity $e$. First, we jump to $a_{1}=a(\xi)$. Then, to $a_{2}=a_{1} \cdot a\left(a_{1}^{-1} \xi\right)$. Then, to $a_{3}=a_{2} \cdot a\left(a_{2}^{-1} \xi\right)$, and so on. To this process correspond nested sets

$$
\Lambda(\xi)=\Lambda_{0}(\xi) \subset \Lambda_{1}(\xi)=a_{1} \Lambda\left(a_{1}^{-1} \xi\right) \subset \Lambda_{2}(\xi)=a_{2} \Lambda\left(a_{2}^{-1} \xi\right) \subset \ldots
$$

covering more and more the group. The successive boundaries of these sets form a sequence of barriers between $e$ and $\xi$. Let also $\Lambda_{k}^{\prime}(\xi)=a_{k} \Lambda^{\prime}\left(a_{k}^{-1} \xi\right)$. The complements of $\Lambda_{i}(\xi)$ and $\Lambda_{i}^{\prime}(\xi)$ are essentially horoballs centered at $\pi_{B} \xi$, at distance respectively $i N+N / 4$ and $i N+N / 2$ of $e$.

Let $\mu \in \bar{U}^{+}$. Consider a nonnegative function $u$ on $\Gamma$ which is, on $\Lambda^{\prime}(\xi)$, positive, harmonic, and bounded by a finite linear combination of functions $G_{\mu}\left(\cdot, y_{i}\right)$ with $y_{i} \notin \Lambda^{\prime}(\xi)$. It is classical that such a function satisfies

$$
u(x)=\sum_{y \notin \Lambda^{\prime}(\xi)} G_{\mu}\left(x, y ; \Lambda^{\prime}(\xi)\right) u(y) .
$$

Indeed, by harmonicity, $u(x)=\sum_{y} \mu\left(x^{-1} y\right) u(y)$. One can then apply again this formula to all the $y$ which are still in $\Lambda^{\prime}(\xi)$, and repeat this algorithm up to time $n$. If $\mu$ is a probability measure, this amounts to 
considering the random walk starting from $x$, stopped once it exits $\Lambda^{\prime}(\xi)$, and saying that the average value of $u$ along this process does not change by harmonicity. We get a formula $u(x)=\sum_{y} F_{n}(y) u(y)$, where for $y \notin \Lambda^{\prime}(\xi)$

$$
F_{n}(y)=\sum_{i \leqslant n} \sum_{\substack{x_{0}=x, x_{1}, \ldots, x_{i}=y \\ x_{1}, \ldots, x_{i-1} \in \Lambda^{\prime}(\xi)}} \mu\left(x_{0}^{-1} x_{1}\right) \cdots \mu\left(x_{i-1}^{-1} x_{i}\right)
$$

converges to $G_{\mu}\left(x, y ; \Lambda^{\prime}(\xi)\right)$ when $n$ tends to $\infty$. On the other hand, for $y \in \Lambda^{\prime}(\xi)$,

$$
F_{n}(y)=\sum_{\substack{x_{0}=x, x_{1}, \ldots, x_{n}=y \\ x_{1}, \ldots, x_{n-1} \in \Lambda^{\prime}(\xi)}} \mu\left(x_{0}^{-1} x_{1}\right) \cdots \mu\left(x_{n-1}^{-1} x_{n}\right) .
$$

One should show that the contribution of these points to the equality $u(x)=\sum_{y} F_{n}(y) u(y)$ tends to 0 when $n$ tends to $\infty$. This follows from the domination condition $u(x) \leqslant C \sum G_{\mu}\left(x, y_{i}\right)$, since this contribution is then bounded by the tails of the series defining the Green function, which tend to 0 as the series is finite.

Remark 4.5. The lifted Martin kernel $K_{\mu}^{B}(\cdot, \xi)=K_{\mu}\left(\cdot, \pi_{B} \xi\right)$ is bounded on $\Lambda^{\prime}(\xi)$ by $C G_{\mu}\left(\cdot, y_{0}\right)$ for any $y_{0} \notin \Lambda^{\prime}(\xi)$, see Remark 4.4. Hence, it satisfies (4.2).

In the formula (4.2), the points $y \notin \Lambda^{\prime}(\xi)$ with a nonzero coefficient $G_{\mu}\left(x, y ; \Lambda^{\prime}(\xi)\right)$ are within bounded distance of $\Lambda^{\prime}(\xi)$, as the walk has bounded jumps. In particular, if $N$ is large enough, they are all included in $\Lambda_{1}(\xi)$. Thus, this formula can also be written as

$$
u(x)=\sum_{y \in \Lambda_{1}(\xi) \backslash \Lambda^{\prime}(\xi)} G_{\mu}\left(x, y ; \Lambda^{\prime}(\xi)\right) u(y) .
$$

This shows how the values of $u$ on $\Lambda_{0}(\xi)$ are determined by its values on $\Lambda_{1}(\xi)$. This formula entails that harmonic functions are fixed point of an operator, which has contracting properties thanks to the good behavior of the kernel $G_{\mu}\left(x, y ; \Lambda^{\prime}(\xi)\right)$ coming from Ancona inequalities. Ultimately, this should provide a tractable analytic characterization of the Martin kernel.

We convert this heuristic discussion into a true operator $\mathcal{M}_{\mu}$, defined for any $\mu \in \bar{U}$. It acts on scalar-valued functions $f(x, \xi)$ defined on pairs $(x, \xi)$ with $\xi \in \partial_{B} \Gamma$ and $x \in \Lambda(\xi)$, by the formula

$$
\mathcal{M}_{\mu} f(x, \xi)=\sum_{y \in \Lambda_{1}(\xi) \backslash \Lambda^{\prime}(\xi)} G_{\mu}\left(x, y ; \Lambda^{\prime}(\xi)\right) f\left(a(\xi)^{-1} y, a(\xi)^{-1} \xi\right) .
$$

Note that the right-hand side is well defined as the point $\left(a(\xi)^{-1} y, a(\xi)^{-1} \xi\right)$ belongs to the domain of definition of $f$, i.e., $a(\xi)^{-1} y \in \Lambda\left(a(\xi)^{-1} \xi\right)$, thanks to the condition $y \in \Lambda_{1}(\xi)$.

To get a fixed point, we should projectivize this operator, normalizing for instance so that the value at $(e, \xi)$ is always 1 . Hence, let

$$
\mathcal{L}_{\mu} f(x, \xi)=\mathcal{M}_{\mu} f(x, \xi) / \mathcal{M}_{\mu} f(e, \xi) .
$$

This is not defined when $\mathcal{M}_{\mu} f(e, \xi)=0$ for some $\xi$. We should also ensure that the sums in the definition of $\mathcal{M}_{\mu}$ are finite.

The contraction properties of this operator are central to the proof of strong Ancona inequalities (Proposition 4.2): For $\mu \in \bar{U}^{+}$, these inequalities are obtained by letting this operator act on a cone of 
positive functions, endowed with a Hilbert distance. To apply the implicit function theorem, we will rather need contraction on a Banach space, that we will deduce from Proposition 4.2. Hence, we will not reprove Proposition 4.2, but rather use its results to obtain another form of contraction.

The iterates of $\mathcal{M}_{\mu}$ (whence of $\mathcal{L}_{\mu}$ ) have the same form. Indeed,

$$
\begin{aligned}
\mathcal{M}_{\mu}^{2} f(x, \xi)= & \sum_{y \notin \Lambda^{\prime}(\xi)} G_{\mu}\left(x, y ; \Lambda^{\prime}(\xi)\right) \mathcal{M}_{\mu} f\left(a(\xi)^{-1} y, a(\xi)^{-1} \xi\right) \\
= & \sum_{y \notin \Lambda^{\prime}(\xi)} G_{\mu}\left(x, y ; \Lambda^{\prime}(\xi)\right) \sum_{z \notin \Lambda^{\prime}\left(a(\xi)^{-1} \xi\right)} G_{\mu}\left(a(\xi)^{-1} y, z ; \Lambda^{\prime}\left(a(\xi)^{-1} \xi\right)\right) \times \\
& \times f\left(a\left(a(\xi)^{-1} \xi\right)^{-1} z, a\left(a(\xi)^{-1} \xi\right)^{-1} a(\xi)^{-1} \xi\right) .
\end{aligned}
$$

Let $w=a(\xi) z$, it belongs to the complement of $\Lambda_{1}^{\prime}(\xi)$. Moreover,

$$
G_{\mu}\left(a(\xi)^{-1} y, z ; \Lambda^{\prime}\left(a(\xi)^{-1} \xi\right)\right)=G_{\mu}\left(y, w ; \Lambda_{1}^{\prime}(\xi)\right) .
$$

Decomposing a trajectory from $x \in \Lambda(\xi)$ to $w$ according to the first point where it exits $\Lambda^{\prime}(\xi)$, we have

$$
\sum_{y \notin \Lambda^{\prime}(\xi)} G_{\mu}\left(x, y ; \Lambda^{\prime}(\xi)\right) G_{\mu}\left(y, w ; \Lambda_{1}^{\prime}(\xi)\right)=G_{\mu}\left(x, w ; \Lambda_{1}^{\prime}(\xi)\right) .
$$

Recalling that $a_{2}(\xi)=a(\xi) \cdot a\left(a(\xi)^{-1} \xi\right)$, the above formula for $\mathcal{M}_{\mu}^{2} f(x, \xi)$ becomes

$$
\mathcal{M}_{\mu}^{2} f(x, \xi)=\sum_{w \notin \Lambda_{1}^{\prime}(\xi)} G_{\mu}\left(x, w ; \Lambda_{1}^{\prime}(\xi)\right) f\left(a_{2}(\xi)^{-1} w, a_{2}(\xi)^{-1} \xi\right) .
$$

The only points with a nonzero coefficient $G_{\mu}\left(x, w ; \Lambda_{1}^{\prime}(\xi)\right)$ belong to $\Lambda_{2}(\xi)$. Hence, the sum may be restricted to $w \in \Lambda_{2}(\xi) \backslash \Lambda_{1}^{\prime}(\xi)$.

In the same way, iterating this argument, one obtains

$$
\mathcal{M}_{\mu}^{n} f(x, \xi)=\sum_{y \in \Lambda_{n}(\xi) \backslash \Lambda_{n-1}^{\prime}(\xi)} G_{\mu}\left(x, y ; \Lambda_{n-1}^{\prime}(\xi)\right) f\left(a_{n}(\xi)^{-1} y, a_{n}(\xi)^{-1} \xi\right) .
$$

Finally, as the projectivization commutes with the iteration of $\mathcal{M}_{\mu}$,

$$
\mathcal{L}_{\mu}^{n} f(x, \xi)=\mathcal{M}_{\mu}^{n} f(x, \xi) / \mathcal{M}_{\mu}^{n} f(e, \xi) .
$$

We define different norms on these functions. Let $\mu \in \bar{U}^{+}$. We set

$$
\|f\|_{\mathcal{C}_{\mu}^{0}}=\sup _{\xi} \sup _{x \in \Lambda(\xi)}|f(x, \xi)| / G_{\mu}(x, e)
$$

and, for small $\beta>0$,

$$
\|f\|_{\mathcal{C}_{\mu}^{\beta}}^{\prime}=\sup _{d\left(\xi, \xi^{\prime}\right) \leqslant e^{-N}} d\left(\xi, \xi^{\prime}\right)^{-\beta} \sup _{x \in \Lambda(\xi)=\Lambda\left(\xi^{\prime}\right)}\left|f(x, \xi)-f\left(x, \xi^{\prime}\right)\right| / G_{\mu}(x, e) .
$$




\section{SÉBASTIEN GOUËZEL}

Finally, let $\|f\|_{\mathcal{C}_{\mu}^{\beta}}=\|f\|_{\mathcal{C}_{\mu}^{0}}+\|f\|_{\mathcal{C}_{\mu}^{\beta}}^{\prime}$. As these are Hölder-like norms, one checks easily that these spaces are Banach algebras: if $f_{1}, f_{2} \in \mathcal{C}_{\mu}^{\beta}$, then $f_{1} f_{2} \in \mathcal{C}_{\mu}^{\beta}$ and

$$
\left\|f_{1} f_{2}\right\|_{\mathcal{C}_{\mu}^{\beta}} \leqslant\left\|f_{1}\right\|_{\mathcal{C}^{\beta}}\left\|f_{2}\right\|_{\mathcal{C}^{\beta}} .
$$

The same holds in $\mathrm{C}_{\mu}^{0}$.

Remark 4.6. Take $x \in \Lambda(\xi)$. Then a geodesics from $x$ to $\pi_{B} \xi$ passes within uniformly bounded distance of $e$. By Ancona inequalities, we obtain $G_{\mu}(x, y) / G_{\mu}(e, y) \asymp G_{\mu}(x, e)$ if $y$ is close to $\pi_{B} \xi$. Letting $y$ tend to $\pi_{B} \xi$ yields

$$
G_{\mu}(x, e) \asymp K_{\mu}^{B}(x, \xi) \quad \text { uniformly in } \xi \in \partial_{B} \Gamma \text { and } x \in \Lambda(\xi) .
$$

Hence, in the definitions of the norms for $\mathcal{C}_{\mu}^{0}$ and $\mathcal{C}_{\mu}^{\beta}$, we could have normalized by $K_{\mu}^{B}(x, \xi)$ instead of $G_{\mu}(x, e)$, obtaining an equivalent norm.

This also implies that, if $d\left(\xi, \xi^{\prime}\right) \leqslant e^{-N}$ (ensuring that $\Lambda(\xi)=\Lambda\left(\xi^{\prime}\right)$ ), then $K_{\mu}^{B}(x, \xi) \asymp K_{\mu}^{B}\left(x, \xi^{\prime}\right)$ uniformly on $x \in \Lambda(\xi)$.

If we want to study a fixed operator $\mathcal{L}_{\mu}$ with $\mu \in \bar{U}^{+}$, the space $\mathcal{C}_{\mu}^{\beta}$ is most natural. However, we want to vary $\mu$ in $U$. Hence, we need a reference space, independent of $\mu$. We recall that we have a measure $\bar{\mu}$ which dominates all measures $\mu \in U$. The spaces $\mathcal{C}_{\mu}^{\beta}$ are all included in $\mathcal{C}_{\bar{\mu}}^{\beta}$. Hence, we can use the latter as a fixed reference space. We will simply write $\mathcal{C}^{0}=\mathcal{C}_{\bar{\mu}}^{0}$ and $\mathcal{C}^{\beta}=\mathcal{C}_{\bar{\mu}}^{\beta}$.

Lemma 4.7. For $\mu \in \bar{U}$ and small enough $\beta$, the operator $\mathcal{M}_{\mu}$ sends continuously $\mathcal{C}^{\beta}$ into itself. If $\mu \in \bar{U}^{+}$, it even sends $\mathrm{e}^{\beta}$ into $\mathrm{C}_{\mu}^{\beta}$. Moreover, the map $(\mu, f) \mapsto \mathcal{M}_{\mu} f$ is analytic from $U \times \mathrm{e}^{\beta}$ to $\mathrm{e}^{\beta}$.

Proof. Consider $f \in \mathrm{e}^{\beta}$ and $\mu \in \bar{U}^{+}$. Then

$$
\begin{aligned}
\left|\mathcal{M}_{\mu} f(x, \xi)\right| & \leqslant \sum_{y \in \Lambda_{1}(\xi) \backslash \Lambda^{\prime}(\xi)} G_{\mu}\left(x, y ; \Lambda^{\prime}(\xi)\right)\left|f\left(a(\xi)^{-1} y, a(\xi)^{-1} \xi\right)\right| \\
& \leqslant \sum_{y \in \Lambda_{1}(\xi) \backslash \Lambda^{\prime}(\xi)} G_{\mu}(x, y) G_{\bar{\mu}}\left(a(\xi)^{-1} y, e\right)\|f\|_{\mathrm{e}^{0}} .
\end{aligned}
$$

As $x \in \Lambda(\xi)$ and $y \notin \Lambda^{\prime}(\xi)$, a geodesics from $x$ to $y$ passes within bounded distance of $e$. Hence, by Ancona inequalities (Lemma 4.3),

$$
G_{\mu}(x, y) \leqslant C G_{\mu}(x, e) G_{\mu}(e, y) \leqslant C G_{\mu}(x, e) G_{\bar{\mu}}(e, y) .
$$

Moreover, $G_{\bar{\mu}}\left(a(\xi)^{-1} y, e\right)=G_{\bar{\mu}}(y, a(\xi)) \leqslant C G_{\bar{\mu}}(y, e)$ as $a(\xi)$ is a bounded distance away from $e$. Hence,

$$
\left|\mathcal{M}_{\mu} f(x, \xi)\right| \leqslant C \sum_{y \in \Gamma} G_{\mu}(x, e) G_{\bar{\mu}}(e, y) G_{\bar{\mu}}(y, e)\|f\|_{\mathcal{\varrho}^{0}} .
$$

Factorizing $G_{\mu}(x, e)\|f\|_{e^{0}}$, we are left with the sum $\sum_{y} G_{\bar{\mu}}(e, y) G_{\bar{\mu}}(y, e)$. Since the spectral radius of $\bar{\mu}$ is $<1$ by construction, the map $r \mapsto G_{r \bar{\mu}}(e, e)$ is well defined and analytic on a neighborhood of 
1. In particular, $G_{\bar{\mu}}(e, e)+\partial G_{r \bar{\mu}}(e, e) /\left.\partial r\right|_{r=1}$ is finite. By an elementary computation (see [GL13b, Proposition 1.9]), this is equal to $\sum_{y} G_{\bar{\mu}}(e, y) G_{\bar{\mu}}(y, e)$. Hence, this sum is finite. This shows that

$$
\left\|\mathcal{M}_{\mu} f\right\|_{\mathcal{C}_{\mu}^{0}} \leqslant C\|f\|_{\mathfrak{e}^{0}}
$$

Let us now control the Hölder norm. Consider $\xi$, $\xi^{\prime}$, write $d\left(\xi, \xi^{\prime}\right)=e^{-n}$. If $n \leqslant 2 N$, then

$$
\begin{aligned}
\left|\mathcal{M}_{\mu} f(x, \xi)-\mathcal{M}_{\mu} f\left(x, \xi^{\prime}\right)\right| /\left(d\left(\xi, \xi^{\prime}\right)^{\beta} G_{\mu}(x, e)\right) & \leqslant e^{2 N \beta}\left(\left|\mathcal{M}_{\mu} f(x, \xi)\right|+\left|\mathcal{M}_{\mu} f\left(x, \xi^{\prime}\right)\right|\right) / G_{\mu}(x, e) \\
& \leqslant C e^{2 N \beta}\|f\|_{e^{0}},
\end{aligned}
$$

by the sup norm control we have already proved. Assume now that $n>2 N$. This entails $\Lambda(\xi)=\Lambda\left(\xi^{\prime}\right)$, and $a(\xi)=a\left(\xi^{\prime}\right)$, and $\Lambda\left(a(\xi)^{-1} \xi\right)=\Lambda\left(a\left(\xi^{\prime}\right)^{-1} \xi^{\prime}\right)$. Then, for $x \in \Lambda(\xi)=\Lambda\left(\xi^{\prime}\right)$,

$$
\begin{aligned}
\mid \mathcal{M}_{\mu} f(x, \xi) & -\mathcal{M}_{\mu} f\left(x, \xi^{\prime}\right) \mid \\
& =\left|\sum_{y \in \Lambda_{1}(\xi) \backslash \Lambda^{\prime}(\xi)} G_{\mu}\left(x, y ; \Lambda^{\prime}(\xi)\right)\left(f\left(a(\xi)^{-1} y, a(\xi)^{-1} \xi\right)-f\left(a\left(\xi^{\prime}\right)^{-1} y, a\left(\xi^{\prime}\right)^{-1} \xi^{\prime}\right)\right)\right| \\
& \leqslant \sum_{y \in \Lambda_{1}(\xi) \backslash \Lambda^{\prime}(\xi)} G_{\mu}(x, y) d\left(a(\xi)^{-1} \xi, a(\xi)^{-1} \xi^{\prime}\right)^{\beta} G_{\bar{\mu}}\left(a(\xi)^{-1} y, e\right)\|f\|_{\mathcal{C}^{\beta}}^{\prime} .
\end{aligned}
$$

As above, this is bounded by

$$
C G_{\mu}(x, e) d\left(a(\xi)^{-1} \xi, a(\xi)^{-1} \xi^{\prime}\right)^{\beta}\|f\|_{e^{\beta}}^{\prime} \leqslant C e^{\beta N} G_{\mu}(x, e) d\left(\xi, \xi^{\prime}\right)^{\beta}\|f\|_{e^{\beta}}^{\prime}
$$

by (3.3). This shows that $\mathcal{M}_{\mu}$ maps continuously $\mathcal{C}^{\beta}$ into $\mathcal{C}_{\mu}^{\beta}$ when $\mu \in \bar{U}^{+}$.

When $\mu \in U$, we show in the same way that $\mathcal{M}_{\mu}$ maps continuously $\mathcal{C}^{\beta}$ into itself. The computation is the same, excepted that $G_{\mu}$ is not positive any more. Hence, one should bound $\left|G_{\mu}(x, y)\right|$ by $G_{\bar{\mu}}(x, y)$ at the beginning of the computation, and then proceed in the same way.

The map $F:(\mu, f) \mapsto \mathcal{M}_{\mu} f$ on $\mathrm{e}^{\beta}$ is linear in $f$. To prove that it is analytic, we should control its dependence on $\mu$. Each function $\mu \mapsto G_{\mu}\left(x, y ; \Lambda^{\prime}(\xi)\right)$ is a limit of a degree $K$ polynomial, obtained by considering the weight of trajectories of length at most $K$. Moreover, the corresponding $K$-truncated operators all satisfy the same bounds as $F$, since the above computations apply. Hence, $F$ is the uniform limit of analytic operators $F_{K}$. As analyticity is stable under uniform convergence (see Paragraph 2.3), it follows that $F$ itself is analytic.

Corollary 4.8. Let $\mu_{1} \in U$ and $f_{1} \in \mathfrak{e}^{\beta}$ satisfy $\mathcal{M}_{\mu_{1}} f_{1}(e, \xi) \neq 0$ for all $\xi$. Then the operator $(\mu, f) \mapsto \mathcal{L}_{\mu} f$ is well defined and analytic from a neighborhood of $\left(\mu_{1}, f_{1}\right)$ in $U \times \mathrm{C}^{\beta}$, to $\mathrm{C}^{\beta}$.

Proof. The operator to be studied is the composition of the operators $(\mu, f) \mapsto \mathcal{M}_{\mu} f$ and $\mathcal{N}: f \mapsto$ $\tilde{f}(x, \xi)=f(x, \xi) / f(e, \xi)$. The first one is well defined and analytic by Lemma 4.7. Hence, it suffices to show that $\mathcal{N}$ is well defined and analytic on the open set $\mathcal{D}^{\beta} \subseteq \mathcal{C}^{\beta}$ of functions $f$ with $f(e, \xi) \neq 0$ for all $\xi$. 


\section{SÉBASTIEN GOUËZEL}

Let us first check that, if $f \in \mathcal{D}^{\beta}$, then $\mathcal{N} f \in \mathcal{C}^{\beta}$. The supremum condition is obvious since $1 /|f(e, \xi)|$ is uniformly bounded, by compactness of $\partial_{B} \Gamma$ and continuity. We check the Hölder condition. We have

$$
\begin{aligned}
\left|\mathcal{N} f(x, \xi)-\mathcal{N} f\left(x, \xi^{\prime}\right)\right| & =\left|f(x, \xi) / f(e, \xi)-f\left(x, \xi^{\prime}\right) / f\left(e, \xi^{\prime}\right)\right| \\
& \leqslant C\left|f(x, \xi) f\left(e, \xi^{\prime}\right)-f(e, \xi) f\left(x, \xi^{\prime}\right)\right| \\
& \leqslant C\left|f(x, \xi)-f\left(x, \xi^{\prime}\right)\right|\left|f\left(e, \xi^{\prime}\right)\right|+C\left|f\left(x, \xi^{\prime}\right)\right|\left|f\left(e, \xi^{\prime}\right)-f(e, \xi)\right|
\end{aligned}
$$

The first term is bounded by $C d\left(\xi, \xi^{\prime}\right)^{\beta} G_{\bar{\mu}}(x, e)$. In the second one, we have $\left|f\left(x, \xi^{\prime}\right)\right| \leqslant C G_{\bar{\mu}}(x, e)$ and $\left|f\left(e, \xi^{\prime}\right)-f(e, \xi)\right| \leqslant C d\left(\xi^{\prime}, \xi\right)^{\beta}$, hence we obtain the same bound. This shows that $\mathcal{N} f \in \mathcal{C}^{\beta}$.

For the analyticity, let us fix $f \in \mathcal{D}^{\beta}$. For small $h \in \mathfrak{C}^{\beta}$,

$$
\mathcal{N}(f+h)(x, \xi)=\frac{(f+h)(x, \xi)}{(f+h)(e, \xi)}=\frac{(f+h)(x, \xi)}{f(e, \xi)} \sum(-1)^{n}(h / f)(e, \xi)^{n} .
$$

This power series converges on a ball with positive radius, as $\mathrm{e}^{\beta}$ is a Banach algebra.

In the same way, one proves the following:

Corollary 4.9. Let $\mu \in \bar{U}^{+}$and $f_{1} \in \mathcal{C}^{\beta}$ satisfy $\mathcal{M}_{\mu} f_{1}(e, \xi) \neq 0$ for all $\xi$. Then the operator $f \mapsto \mathcal{L}_{\mu} f$ is well defined and analytic from a neighborhood of $f_{1}$ in $\mathrm{e}^{\beta}$, to $\mathrm{C}_{\mu}^{\beta}$.

Proof. The operator $\mathcal{L}_{\mu}$ is the composition of $\mathcal{M}_{\mu}$ and $\mathcal{N}: f \mapsto \tilde{f}(x, \xi)=f(x, \xi) / f(e, \xi)$. The first one is linear from $\mathcal{C}^{\beta}$ to $\mathcal{C}_{\mu}^{\beta}$ by Lemma 4.7, the second one is analytic as we explained in the proof of Corollary 4.8 (on $\mathcal{C}^{\beta}$, but the same proof works in $\mathcal{C}_{\mu}^{\beta}$ ). Hence, their composition is analytic.

Lemma 4.10. For small enough $\beta$ and for $\mu \in \bar{U}^{+}$, the function $(x, \xi) \mapsto K_{\mu}^{B}(x, \xi)$ belongs to $\mathcal{C}^{\beta}$.

Proof. By (4.6), $K_{\mu}^{B}(x, \xi) \asymp G_{\mu}(x, e)$ uniformly in $\xi \in \partial_{B} \Gamma$ and $x \in \Lambda(\xi)$. Moreover, $G_{\mu}(x, e) \leqslant G_{\bar{\mu}}(x, e)$. This proves that $\left\|K_{\mu}^{B}\right\|_{\mathrm{e}^{0}}<\infty$.

Let us estimate its Hölder norm. Consider $\xi$, $\xi^{\prime}$ with $d\left(\xi, \xi^{\prime}\right)=e^{-n}$ for some $n \geqslant N$. Proposition 4.2 applies to Martin kernels, by Remark 4.4. It shows that, for any $x \in \Lambda(\xi)$,

$$
\left|\frac{K_{\mu}^{B}(x, \xi) / K_{\mu}^{B}(e, \xi)}{K_{\mu}^{B}\left(x, \xi^{\prime}\right) / K_{\mu}^{B}\left(e, \xi^{\prime}\right)}-1\right| \leqslant C e^{-C^{-1} n} .
$$

As $K_{\mu}^{B}(e, \xi)=K_{\mu}^{B}\left(e, \xi^{\prime}\right)=1$, we obtain

$$
\left|K_{\mu}^{B}(x, \xi)-K_{\mu}^{B}\left(x, \xi^{\prime}\right)\right| \leqslant K_{\mu}^{B}\left(x, \xi^{\prime}\right) C e^{-C^{-1} n} .
$$

The term on the right hand side is bounded by $C^{\prime} G_{\bar{\mu}}(x, e) d\left(\xi, \xi^{\prime}\right)^{\beta}$ if $\beta$ is small enough.

The operators $\mathcal{L}_{\mu}$ were defined precisely so that the following lemma holds.

Lemma 4.11. Let $\mu \in \bar{U}^{+}$. The function $(x, \xi) \mapsto K_{\mu}^{B}(x, \xi)$ is a fixed point of $\mathcal{L}_{\mu}$. It is the only one among positive functions in $\mathrm{C}^{\beta}$. 


\section{ANALYTICITY OF THE ENTROPy AND THE EsCAPE RATE}

Proof. As $K_{\mu}^{B} \in \mathcal{C}^{\beta}$ by Lemma 4.10 , the operator $\mathcal{M}_{\mu}$ is well defined on $K_{\mu}^{B}$. By positivity, $\mathcal{L}_{\mu} K_{\mu}^{B}$ is also well defined.

By Remark 4.5, the function $K_{\mu}^{B}$ satisfies the equation (4.2), i.e.,

$$
K_{\mu}^{B}(x, \xi)=\sum_{y \notin \Lambda^{\prime}(\xi)} G_{\mu}\left(x, y ; \Lambda^{\prime}(\xi)\right) K_{\mu}^{B}(y, \xi) .
$$

It follows from its definition that $K_{\mu}^{B}$ satisfies the following multiplicative cocycle equation:

$$
K_{\mu}^{B}(y, \xi)=K_{\mu}^{B}\left(a^{-1} y, a^{-1} \xi\right) / K_{\mu}^{B}\left(a^{-1}, a^{-1} \xi\right) .
$$

Therefore, (4.5) gives

$$
K_{\mu}^{B}(x, \xi)=\sum_{y \notin \Lambda^{\prime}(\xi)} G_{\mu}\left(x, y ; \Lambda^{\prime}(\xi)\right) K_{\mu}^{B}\left(a(\xi)^{-1} y, a(\xi)^{-1} \xi\right) / K_{\mu}^{B}\left(a(\xi)^{-1}, a(\xi)^{-1} \xi\right) .
$$

Hence,

$$
\mathcal{M}_{\mu} K_{\mu}^{B}(x, \xi)=K_{\mu}^{B}\left(a(\xi)^{-1}, a(\xi)^{-1} \xi\right) K_{\mu}^{B}(x, \xi) .
$$

Applying this equation to $x=e$ and dividing both sides, we get $K_{\mu}^{B}(x, \xi) / K_{\mu}^{B}(e, \xi)=\mathcal{L}_{\mu} K_{\mu}^{B}(x, \xi)$. As $K_{\mu}^{B}(e, \xi)=1$, this concludes the proof that $K_{\mu}^{B}$ is a fixed point of $\mathcal{L}_{\mu}$.

Consider now any fixed point $f>0$ of $\mathcal{L}_{\mu}$ in $\mathcal{C}^{\beta}$. Using the equality (4.4), we get

$$
f(x, \xi)=\mathcal{L}_{\mu}^{n} f(x, \xi)=\mathcal{M}_{\mu}^{n} f(e, \xi)^{-1} \cdot \sum_{y \notin \Lambda_{n-1}^{\prime}} G_{\mu}\left(\xi, y ; \Lambda_{n-1}^{\prime}(\xi)\right) f\left(a_{n}(\xi)^{-1} y, a_{n}(\xi)^{-1} \xi\right) .
$$

Proposition 4.2 yields, for all $x \in \Lambda(\xi)$ and all $y \notin \Lambda_{n-1}^{\prime}(\xi)$ with $G_{\mu}\left(e, y ; \Lambda_{n-1}^{\prime}(\xi)\right)>0$

$$
\left|\frac{G_{\mu}\left(x, y ; \Lambda_{n-1}^{\prime}(\xi)\right) / G_{\mu}\left(e, y ; \Lambda_{n-1}^{\prime}(\xi)\right)}{K_{\mu}^{B}(x, \xi) / K_{\mu}^{B}(e, \xi)}-1\right| \leqslant C e^{-C^{-1} n} .
$$

Let $\varepsilon>0$. For large enough $n$, we obtain

$$
G_{\mu}\left(x, y ; \Lambda_{n-1}^{\prime}(\xi)\right)=(1 \pm \varepsilon) G_{\mu}\left(e, y ; \Lambda_{n-1}^{\prime}(\xi)\right) K_{\mu}^{B}(x, \xi) .
$$

Injecting this estimate into (4.7) (and using the nonnegativity of $f$ ), we get

$$
\begin{aligned}
f(x, \xi) & =(1 \pm \varepsilon) \mathcal{M}_{\mu}^{n} f(e, \xi)^{-1} \cdot \sum_{y \notin \Lambda_{n-1}^{\prime}(\xi)} G_{\mu}\left(e, y ; \Lambda_{n-1}^{\prime}(\xi)\right) K_{\mu}^{B}(x, \xi) f\left(a_{n}(\xi)^{-1} y, a_{n}(\xi)^{-1} \xi\right) \\
& =(1 \pm \varepsilon) \mathcal{M}_{\mu}^{n} f(e, \xi)^{-1} \cdot K_{\mu}^{B}(x, \xi) \mathcal{M}_{\mu}^{n} f(e, \xi)=(1 \pm \varepsilon) K_{\mu}^{B}(x, \xi) .
\end{aligned}
$$

Letting $\varepsilon$ tend to 0 , we obtain $f=K_{\mu}^{B}$.

The following lemma encompasses the contraction properties of $\mathcal{L}_{\mu}$ on $\mathcal{C}^{\beta}$. It is the main technical tool to be able to apply the implicit function theorem later on. 
Lemma 4.12. Assume that $\beta$ is small enough. Let $\mu \in \bar{U}^{+}$. The differential of $\mathcal{L}_{\mu}$ at $K_{\mu}^{B}$ satisfies

$$
\left\|D \mathcal{L}_{\mu}^{n}\left(K_{\mu}^{B}\right): \mathrm{e}^{\beta} \rightarrow \mathrm{e}^{\beta}\right\| \leqslant C e^{-\rho n},
$$

for some $\rho>0$, some $C>0$ and all $n \geqslant 0$.

Proof. Denoting by $I$ the inclusion of $\mathcal{C}_{\mu}^{\beta}$ in $\mathcal{C}^{\beta}$, we have $D \mathcal{L}_{\mu}^{n+1}\left(K_{\mu}^{B}\right)=I \circ D \mathcal{L}_{\mu}^{n}\left(K_{\mu}^{B}\right) \circ D \mathcal{L}_{\mu}\left(K_{\mu}^{B}\right)$ where the operator on the right is well defined and maps continuously $\mathcal{C}^{\beta}$ into $\mathcal{C}_{\mu}^{\beta}$ by Corollary 4.9. Thus, it suffices to show that

$$
\left\|D \mathcal{L}_{\mu}^{n}\left(K_{\mu}^{B}\right): \mathcal{C}_{\mu}^{\beta} \rightarrow \mathcal{C}_{\mu}^{\beta}\right\| \leqslant C e^{-\rho n} .
$$

For ease of notations, we will write $\mathbf{x}$ for a pair $(x, \xi)$, and $e_{\mathbf{x}}=(e, \xi)$. By Remark 4.6, we can define equivalent norms on $\mathcal{C}_{\mu}^{0}$ and $\mathcal{C}_{\mu}^{\beta}$ by

$$
\|f\|_{\overline{\mathcal{e}}_{\mu}^{0}}=\sup _{\mathbf{x}}|f(\mathbf{x})| / K_{\mu}^{B}(\mathbf{x})
$$

and

$$
\|f\|_{\overline{\mathcal{C}}_{\mu}^{\beta}}^{\prime}=\sup _{d\left(\xi, \xi^{\prime}\right) \leqslant e^{-N}} \sup _{x \in \Lambda(\xi)} d\left(\xi, \xi^{\prime}\right)^{-\beta}\left|f(x, \xi)-f\left(x, \xi^{\prime}\right)\right| / K_{\mu}^{B}(x, \xi) .
$$

In this last equation, we could have use $K_{\mu}^{B}(x, \xi)$ or $K_{\mu}^{B}\left(x, \xi^{\prime}\right)$ since their ratio is bounded, again by Remark 4.6. It suffices to prove the inequality (4.8) for the norm $\overline{\mathrm{C}}_{\mu}^{\beta}$, which is equivalent to the original one.

We have $\mathcal{L}_{\mu}^{n} f(\mathbf{x})=\mathcal{M}_{\mu}^{n} f(\mathbf{x}) / \mathcal{M}_{\mu}^{n} f\left(e_{\mathbf{x}}\right)$. Consequently,

$$
D \mathcal{L}_{\mu}^{n}\left(K_{\mu}^{B}\right) f(\mathbf{x})=\frac{\mathcal{M}_{\mu}^{n} f(\mathbf{x}) \cdot \mathcal{M}_{\mu}^{n} K_{\mu}^{B}\left(e_{\mathbf{x}}\right)-\mathcal{M}_{\mu}^{n} f\left(e_{\mathbf{x}}\right) \mathcal{M}_{\mu}^{n} K_{\mu}^{B}(\mathbf{x})}{\left(\mathcal{M}_{\mu}^{n} K_{\mu}^{B}\left(e_{\mathbf{x}}\right)\right)^{2}} .
$$

By (4.4), the operator $\mathcal{M}_{\mu}^{n}$ can be written as

$$
\mathcal{M}_{\mu}^{n} f(\mathbf{x})=\sum_{\mathbf{y}} F_{n}(\mathbf{x}, \mathbf{y}) f(\mathbf{y})
$$

where the kernel $F_{n}$ is a relative Green function, given by

$$
F_{n}((x, \xi),(y, \eta))=1_{\eta=a_{n}(\xi)^{-1} \xi} 1_{a_{n}(\xi) y \in \Lambda_{n}(\xi) \backslash \Lambda_{n-1}^{\prime}(\xi)} G_{\mu}\left(x, a_{n}(\xi) y ; \Lambda_{n-1}^{\prime}(\xi)\right) .
$$

When we write $F_{n}((x, \xi),(y, \eta))$, we will implicitly only consider those pairs where $F_{n}$ can be nonzero, i.e., those where $\eta=a_{n}(\xi)^{-1} \xi$ and $a_{n}(\xi) y \in \Lambda_{n}(\xi) \backslash \Lambda_{n-1}^{\prime}(\xi)$.

The following property of $F_{n}$ follows from Proposition 4.2: There exist $\rho_{0}>0$ and $C>0$ such that, for all $\mathbf{x}$, and all $\mathbf{y}, \mathbf{z}$ with $F_{n}(\mathbf{x}, \mathbf{y}) \neq 0, F_{n}(\mathbf{x}, \mathbf{z}) \neq 0$,

$$
\left|\frac{F_{n}(\mathbf{x}, \mathbf{z}) / F_{n}\left(e_{\mathbf{x}}, \mathbf{z}\right)}{F_{n}(\mathbf{x}, \mathbf{y}) / F_{n}\left(e_{\mathbf{x}}, \mathbf{y}\right)}-1\right| \leqslant C e^{-\rho_{0} n} .
$$

In particular,

$$
\left|F_{n}\left(e_{\mathbf{x}}, \mathbf{y}\right) F_{n}(\mathbf{x}, \mathbf{z})-F_{n}(\mathbf{x}, \mathbf{y}) F_{n}\left(e_{\mathbf{x}}, \mathbf{z}\right)\right| \leqslant C e^{-\rho_{0} n} F_{n}(\mathbf{x}, \mathbf{y}) F_{n}\left(e_{\mathbf{x}}, \mathbf{z}\right) .
$$


By (4.9),

$$
D \mathcal{L}_{\mu}^{n}\left(K_{\mu}^{B}\right) f(\mathbf{x})=\frac{\sum_{\mathbf{y}, \mathbf{z}}\left(F_{n}(\mathbf{x}, \mathbf{y}) F_{n}\left(e_{\mathbf{x}}, \mathbf{z}\right)-F_{n}\left(e_{\mathbf{x}}, \mathbf{y}\right) F_{n}(\mathbf{x}, \mathbf{z})\right) f(\mathbf{y}) K_{\mu}^{B}(\mathbf{z})}{\left(\sum_{\mathbf{y}} F_{n}\left(e_{\mathbf{x}}, \mathbf{y}\right) K_{\mu}^{B}(\mathbf{y})\right)^{2}} .
$$

With (4.11), we get

$$
\left|D \mathcal{L}_{\mu}^{n}\left(K_{\mu}^{B}\right) f(\mathbf{x})\right| \leqslant C e^{-\rho_{0} n} \frac{\sum_{\mathbf{y}, \mathbf{z}} F_{n}(\mathbf{x}, \mathbf{y}) F_{n}\left(e_{\mathbf{x}}, \mathbf{z}\right)|f(\mathbf{y})| K_{\mu}^{B}(\mathbf{z})}{\left(\sum_{\mathbf{y}} F_{n}\left(e_{\mathbf{x}}, \mathbf{y}\right) K_{\mu}^{B}(\mathbf{y})\right)^{2}} .
$$

The sum on the numerator can be factored. The part with $\mathbf{z}$ cancels out one of the factors of the denominator. In the $\mathbf{y}$ part, we have by definition $|f(\mathbf{y})| \leqslant\|f\|_{\bar{e}_{\mu}^{0}} K_{\mu}^{B}(\mathbf{y})$. Hence,

$$
\begin{aligned}
\left|D \mathcal{L}_{\mu}^{n}\left(K_{\mu}^{B}\right) f(\mathbf{x})\right| & \leqslant C\|f\|_{\overline{\mathcal{C}}_{\mu}^{0}} e^{-\rho_{0} n} \frac{\sum_{\mathbf{y}} F_{n}(\mathbf{x}, \mathbf{y}) K_{\mu}^{B}(\mathbf{y})}{\sum_{\mathbf{y}} F_{n}\left(e_{\mathbf{x}}, \mathbf{y}\right) K_{\mu}^{B}(\mathbf{y})}=C\|f\|_{\bar{e}_{\mu}^{0}} e^{-\rho_{0} n} \mathcal{L}_{\mu}^{n} K_{\mu}^{B}(\mathbf{x}) \\
& =C\|f\|_{\overline{\mathcal{C}}_{\mu}^{0}} e^{-\rho_{0} n} K_{\mu}^{B}(\mathbf{x})
\end{aligned}
$$

as $K_{\mu}^{B}$ is a fixed point of $\mathcal{L}_{\mu}$. This shows that $\left\|D \mathcal{L}_{\mu}^{n}\left(K_{\mu}^{B}\right) f\right\|_{\overline{\mathcal{C}}_{\mu}^{0}} \leqslant C e^{-\rho_{0} n}\|f\|_{\overline{\mathcal{C}}_{\mu}^{0}}$, as claimed.

Let us now control its Hölder norm. Let $\mathbf{x}=(x, \xi)$ and $\mathbf{x}^{\prime}=\left(x, \xi^{\prime}\right)$, with $d\left(\xi, \xi^{\prime}\right)=e^{-k}$ for some $k \geqslant N$. We should bound $\left|D \mathcal{L}_{\mu}^{n}\left(K_{\mu}^{B}\right) f(\mathbf{x})-D \mathcal{L}_{\mu}^{n}\left(K_{\mu}^{B}\right) f\left(\mathbf{x}^{\prime}\right)\right| /\left(d\left(\xi, \xi^{\prime}\right)^{\beta} K_{\mu}^{B}(\mathbf{x})\right)$.

If $k \leqslant(n+1) N$, we write

$$
\begin{aligned}
\left|D \mathcal{L}_{\mu}^{n}\left(K_{\mu}^{B}\right) f(\mathbf{x})-D \mathcal{L}_{\mu}^{n}\left(K_{\mu}^{B}\right) f\left(\mathbf{x}^{\prime}\right)\right| & \leqslant\left|D \mathcal{L}_{\mu}^{n}\left(K_{\mu}^{B}\right) f(\mathbf{x})\right|+\left|D \mathcal{L}_{\mu}^{n}\left(K_{\mu}^{B}\right) f\left(\mathbf{x}^{\prime}\right)\right| \\
& \leqslant\left\|D \mathcal{L}_{\mu}^{n}\left(K_{\mu}^{B}\right) f\right\|_{\overline{\mathcal{C}}_{\mu}^{0}}\left(K_{\mu}^{B}(\mathbf{x})+K_{\mu}^{B}\left(\mathbf{x}^{\prime}\right)\right) \\
& \leqslant C\left\|D \mathcal{L}_{\mu}^{n}\left(K_{\mu}^{B}\right) f\right\|_{\overline{\mathcal{C}}_{\mu}^{0}} K_{\mu}^{B}(\mathbf{x}),
\end{aligned}
$$

where the last inequality uses the fact that $K_{\mu}^{B}\left(\mathbf{x}^{\prime}\right) \leqslant C K_{\mu}^{B}(\mathbf{x})$, explained in Remark 4.6. Therefore,

$$
\begin{aligned}
\left|D \mathcal{L}_{\mu}^{n}\left(K_{\mu}^{B}\right) f(\mathbf{x})-D \mathcal{L}_{\mu}^{n}\left(K_{\mu}^{B}\right) f\left(\mathbf{x}^{\prime}\right)\right| /\left(d\left(\xi, \xi^{\prime}\right)^{\beta} K_{\mu}^{B}(\mathbf{x})\right) & \leqslant C e^{\beta(n+1) N}\left\|D \mathcal{L}_{\mu}^{n}\left(K_{\mu}^{B}\right) f\right\|_{\overline{\mathcal{C}}_{\mu}^{0}} \\
& \leqslant C e^{\beta(n+1) N} e^{-\rho_{0} n}\|f\|_{\overline{\mathcal{C}}_{\mu}^{0}}
\end{aligned}
$$

If $\beta$ is small enough (i.e., $\beta<\rho_{0} / N$ ), this is exponentially small as desired.

Assume now that $k>(n+1) N$. Then $a_{n}(\xi)=a_{n}\left(\xi^{\prime}\right)$ (we will simply denote it by $a_{n}$ ). Moreover, the two horofunctions given by $\eta=a_{n}^{-1} \xi$ and $\eta^{\prime}=a_{n}^{-1} \xi^{\prime}$ coincide on the ball of radius $N$. Thus, the summation set in (4.10) is the same for $\mathbf{x}$ and $\mathbf{x}^{\prime}$. Moreover, for any $y$, we have $F_{n}((x, \xi),(y, \eta))=$ $F_{n}\left(\left(x, \xi^{\prime}\right),\left(y, \eta^{\prime}\right)\right)$. Taking $\xi, \xi^{\prime}$ (and therefore $\eta$ and $\eta^{\prime}$ ) as fixed, we will simply write $\tilde{F}_{n}(x, y)$ for this common quantity. Let $u(\eta)=1 /\left(\sum_{y} \tilde{F}_{n}(e, y) K_{\mu}^{B}(y, \eta)\right)^{2}$, and define $u\left(\eta^{\prime}\right)$ in the same way with $\eta^{\prime}$. By (4.12),

$$
D \mathcal{L}_{\mu}^{n}\left(K_{\mu}^{B}\right) f(\mathbf{x})=\sum_{y, z}\left(\tilde{F}_{n}(x, y) \tilde{F}_{n}(e, z)-\tilde{F}_{n}(e, y) \tilde{F}_{n}(x, z)\right) f(y, \eta) K_{\mu}^{B}(z, \eta) u(\eta)
$$




\section{SÉBASTIEN GOUËZEL}

The same formula holds for $\mathbf{x}^{\prime}$. Hence,

$$
\begin{aligned}
& \quad\left|D \mathcal{L}_{\mu}^{n}\left(K_{\mu}^{B}\right) f(\mathbf{x})-D \mathcal{L}_{\mu}^{n}\left(K_{\mu}^{B}\right) f\left(\mathbf{x}^{\prime}\right)\right|= \\
& \quad\left|\sum_{y, z}\left(\tilde{F}_{n}(x, y) \tilde{F}_{n}(e, z)-\tilde{F}_{n}(e, y) \tilde{F}_{n}(x, z)\right) \cdot\left(f(y, \eta) K_{\mu}^{B}(z, \eta) u(\eta)-f\left(y, \eta^{\prime}\right) K_{\mu}^{B}\left(z, \eta^{\prime}\right) u\left(\eta^{\prime}\right)\right)\right| .
\end{aligned}
$$

We use the equality $a b c-a^{\prime} b^{\prime} c^{\prime}=\left(a-a^{\prime}\right) b c+a^{\prime}\left(b-b^{\prime}\right) c+a^{\prime} b^{\prime}\left(c-c^{\prime}\right)$ to bound the last difference. We have

$$
\left|f(y, \eta)-f\left(y, \eta^{\prime}\right)\right| \leqslant d\left(\eta, \eta^{\prime}\right)^{\beta}\|f\|_{\overline{\mathcal{C}}_{\mu}^{\beta}} K_{\mu}^{B}(y, \eta) .
$$

As $K_{\mu}^{B} \in \mathcal{C}_{\mu}^{\beta}=\overline{\mathcal{C}}_{\mu}^{\beta}$ by Lemma 4.10,

$$
\left|K_{\mu}^{B}(z, \eta)-K_{\mu}^{B}\left(z, \eta^{\prime}\right)\right| \leqslant C d\left(\eta, \eta^{\prime}\right)^{\beta} K_{\mu}^{B}(z, \eta) .
$$

Finally, $u(\eta)=1 /\left(\mathcal{M}_{\mu}^{n} K_{\mu}^{B}(e, \xi)\right)^{2}$. By (4.6), this equals $1 / K_{\mu}^{B}\left(a_{n}^{-1}, a_{n}^{-1} \xi\right)^{2}=1 / K_{\mu}^{B}\left(a_{n}^{-1}, \eta\right)^{2}$. We have $\left|K_{\mu}^{B}\left(a_{n}^{-1}, \eta\right)-K_{\mu}^{B}\left(a_{n}^{-1}, \eta^{\prime}\right)\right| \leqslant C d\left(\eta, \eta^{\prime}\right)^{\beta} K_{\mu}^{B}\left(a_{n}^{-1}, \eta\right)$ since $K_{\mu}^{B} \in \mathcal{C}_{\mu}^{\beta}=\overline{\mathcal{C}}_{\mu}^{\beta}$. Moreover, the ratio $K_{\mu}^{B}\left(a_{n}^{-1}, \eta\right) / K_{\mu}^{B}\left(a_{n}^{-1}, \eta^{\prime}\right)$ is uniformly bounded by Remark 4.6. Therefore,

$$
\begin{aligned}
\left|u(\eta)-u\left(\eta^{\prime}\right)\right| & =\left|\frac{\left(K_{\mu}^{B}\left(a_{n}^{-1}, \eta\right)-K_{\mu}^{B}\left(a_{n}^{-1}, \eta^{\prime}\right)\right)\left(K_{\mu}^{B}\left(a_{n}^{-1}, \eta\right)+K_{\mu}^{B}\left(a_{n}^{-1}, \eta^{\prime}\right)\right)}{K_{\mu}^{B}\left(a_{n}^{-1}, \eta\right)^{2} K_{\mu}^{B}\left(a_{n}^{-1}, \eta^{\prime}\right)^{2}}\right| \\
& \leqslant C d\left(\eta, \eta^{\prime}\right)^{\beta} / K_{\mu}^{B}\left(a_{n}^{-1}, \eta\right)^{2}=C d\left(\eta, \eta^{\prime}\right)^{\beta} u(\eta) .
\end{aligned}
$$

Combining these inequalities, we obtain

$$
\left|f(y, \eta) K_{\mu}^{B}(z, \eta) u(\eta)-f\left(y, \eta^{\prime}\right) K_{\mu}^{B}\left(z, \eta^{\prime}\right) u\left(\eta^{\prime}\right)\right| \leqslant C d\left(\eta, \eta^{\prime}\right)^{\beta}\|f\|_{\overline{\mathfrak{e}}_{\mu}^{\beta}} K_{\mu}^{B}(y, \eta) K_{\mu}^{B}(z, \eta) u(\eta) .
$$

Together with (4.13), this yields

$$
\begin{aligned}
\left|D \mathcal{L}_{\mu}^{n}\left(K_{\mu}^{B}\right) f(\mathbf{x})-D \mathcal{L}_{\mu}^{n}\left(K_{\mu}^{B}\right) f\left(\mathbf{x}^{\prime}\right)\right| & \\
& \leqslant C d\left(\eta, \eta^{\prime}\right)^{\beta}\|f\|_{\tilde{\mathcal{C}}_{\mu}^{\beta}} \sum_{y, z}\left|\tilde{F}_{n}(x, y) \tilde{F}_{n}(e, z)-\tilde{F}_{n}(e, y) \tilde{F}_{n}(x, z)\right| K_{\mu}^{B}(y, \eta) K_{\mu}^{B}(z, \eta) u(\eta)
\end{aligned}
$$

The sum is precisely the sum we have handled in the control of the sup norm, in (4.12), with $f$ replaced by $K_{\mu}^{B}$. We have shown that it is bounded by $C e^{-\rho_{0} n} K_{\mu}^{B}(\mathbf{x})$. Finally, we get

$$
\left|D \mathcal{L}_{\mu}^{n}\left(K_{\mu}^{B}\right) f(\mathbf{x})-D \mathcal{L}_{\mu}^{n}\left(K_{\mu}^{B}\right) f\left(\mathbf{x}^{\prime}\right)\right| \leqslant C e^{-\rho_{0} n} d\left(\eta, \eta^{\prime}\right)^{\beta} K_{\mu}^{B}(\mathbf{x})\|f\|_{\overline{\mathcal{C}}_{\mu}^{\beta}}
$$

As $\eta=a_{n}^{-1} \xi$ and $\eta^{\prime}=a_{n}^{-1} \xi^{\prime}$ with $\left|a_{n}\right| \leqslant n N$, we have $d\left(\eta, \eta^{\prime}\right) \leqslant e^{n N} d\left(\xi, \xi^{\prime}\right)$ by (3.3). Therefore,

$$
\left|D \mathcal{L}_{\mu}^{n}\left(K_{\mu}^{B}\right) f(\mathbf{x})-D \mathcal{L}_{\mu}^{n}\left(K_{\mu}^{B}\right) f\left(\mathbf{x}^{\prime}\right)\right| \leqslant C e^{\beta n N} e^{-\rho_{0} n} d\left(\xi, \xi^{\prime}\right)^{\beta} K_{\mu}^{B}(\mathbf{x})\|f\|_{\overline{\mathfrak{C}}_{\mu}^{\beta}} .
$$

If $\beta<\rho_{0} / N$, this is again exponentially small as desired. 
Let us consider the transformation $\mathcal{Q}:(\mu, f) \mapsto \mathcal{L}_{\mu} f-f$, defined on a neighborhood of $\left(\mu_{0}, K_{\mu_{0}}^{B}\right)$ in $U \times \mathcal{C}^{\beta}$ for a suitably small $\beta$. It satisfies $Q\left(\mu_{0}, K_{\mu_{0}}^{B}\right)=0$ as $K_{\mu_{0}}^{B}$ is a fixed point of $\mathcal{L}_{\mu_{0}}$ by Lemma 4.11. Moreover, $\partial_{f} \mathcal{Q}\left(\mu_{0}, K_{\mu_{0}}^{B}\right)=D \mathcal{L}_{\mu_{0}}\left(K_{\mu_{0}}^{B}\right)$ - Id is invertible, by Lemma 4.12. The implicit function theorem, in its analytic version, shows the existence of an analytic family $\mu \mapsto f_{\mu}$, for $\mu$ close to $\mu_{0}$, with $f_{\mu_{0}}=K_{\mu_{0}}^{B}$, and such that $Q\left(\mu, f_{\mu}\right)=0$, i.e., $\mathcal{L}_{\mu} f_{\mu}=f_{\mu}$.

Lemma 4.13. For $\mu \in U^{+}$close enough to $\mu_{0}$, the function $f_{\mu}$ is everywhere positive.

Proof. As $\mu \mapsto f_{\mu}$ is analytic, it is continuous. For $\mu$ close to $\mu_{0}$, the function $f_{\mu}$ is close in $\mathcal{C}^{\beta}$ to $f_{0}=K_{\mu_{0}}^{B}$. As

$$
f_{\mu}(x, \xi)=\mathcal{L}_{\mu} f_{\mu}(x, \xi)=\mathcal{M}_{\mu} f_{\mu}(x, \xi) / \mathcal{M}_{\mu} f_{\mu}(e, \xi),
$$

it suffices to show that $\mathcal{M}_{\mu} f$ is positive if $f$ is close enough to $f_{0}$ in $\mathrm{C}^{\beta}$, and $\mu \in U^{+}$.

Let $\varepsilon>0$. Any $f$ close enough to $f_{0}$ in $\mathcal{C}^{\beta}$ satisfies $f(x, \xi) \geqslant f_{0}(x, \xi)-\varepsilon G_{\bar{\mu}}(x, e)$ by definition of the norm. We obtain

$$
\mathcal{M}_{\mu} f(x, \xi) \geqslant \mathcal{M}_{\mu} f_{0}(x, \xi)-\varepsilon \mathcal{M}_{\mu} G_{\bar{\mu}}(x, e) \geqslant \mathcal{M}_{\mu} f_{0}(x, \xi)-C \varepsilon G_{\mu}(x, e) .
$$

For the last inequality, we used the fact that $(x, \xi) \mapsto G_{\bar{\mu}}(x, e)$ belongs obviously to $\mathcal{C}^{0}$, and the fact that $\mathcal{M}_{\mu}$ maps $\mathrm{C}^{0}$ into $\mathrm{C}_{\mu}^{0}$ by Lemma 4.7 .

Let $\xi \in \partial_{B} \Gamma$. Consider a point $y=y(\xi)$, close to a geodesic from $e$ to $a(\xi)$, which does not belong to $\Lambda^{\prime}(\xi)$ but such that $G_{\mu_{0}}\left(e, y ; \Lambda^{\prime}(\xi)\right)>0$, i.e., $y$ is close enough to the boundary of $\Lambda^{\prime}(\xi)$ to be an exit point of the random walk in $\Lambda^{\prime}(\xi)$. It satisfies $G_{\mu}\left(e, y ; \Lambda^{\prime}(\xi)\right)>0$ as $\mu \geqslant \mu_{0} / 2$ thanks to the definition of $U$. The function $x \mapsto G_{\mu}\left(x, y ; \Lambda^{\prime}(\xi)\right)$ is positive and harmonic on $\Lambda(\xi)$. Therefore, Proposition 4.2 applied to this function and to $x \mapsto G_{\mu}(x, y)$ shows that

$$
\frac{G_{\mu}(x, y) / G_{\mu}(e, y)}{G_{\mu}\left(x, y ; \Lambda^{\prime}(\xi)\right) / G_{\mu}\left(e, y ; \Lambda^{\prime}(\xi)\right)} \leqslant C
$$

uniformly in $x \in \Lambda(\xi)$. The quantities $G_{\mu}\left(e, y ; \Lambda^{\prime}(\xi)\right)$ and $G_{\mu}(e, y)$ are bounded from above and from below, uniformly. We obtain $G_{\mu}\left(x, y ; \Lambda^{\prime}(\xi)\right) \geqslant C^{-1} G_{\mu}(x, y)$, which is $\geqslant C^{-1} G_{\mu}(x, e)$ thanks to Harnack inequalities (as $d(e, y)$ is uniformly bounded).

By the definition (4.3) of $\mathcal{M}_{\mu}$,

$$
\mathcal{M}_{\mu} f_{0}(x, \xi) \geqslant G_{\mu}\left(x, y(\xi) ; \Lambda^{\prime}(\xi)\right) f_{0}\left(a(\xi)^{-1} y, a(\xi)^{-1} \xi\right) \geqslant C^{-1} G_{\mu}(x, e) \cdot C^{-1},
$$

as $f_{0}$ is uniformly bounded from below on points which are a bounded distance away from the identify.

Finally, we obtain from (4.14)

$$
\mathcal{M}_{\mu} f(x, \xi) \geqslant C^{-1} G_{\mu}(x, e)-C \varepsilon G_{\mu}(x, e) .
$$

If $\varepsilon$ is small enough, this is bounded from below by a positive multiple of $G_{\mu}(x, e)$, uniformly in $(x, \xi)$.

We can now prove that the entropy depends analytically on the measure. 
Proof of Theorem 4.1. By Lemma 4.13, there exists a neighborhood $V_{0}$ of $\mu_{0}$ in $U \subseteq \mathcal{P}(F)$ such that, for $\mu \in V_{0} \cap U^{+}$, the function $f_{\mu}$ is a fixed point of $\mathcal{L}_{\mu}$ in $\mathcal{C}^{\beta}$, everywhere positive. By Lemma 4.11, it has to coincide with $K_{\mu}^{B}$. Moreover, $\mu \mapsto f_{\mu}$ is analytic from $V_{0}$ to $\mathrm{e}^{\beta}$ for some $\beta>0$.

We have also constructed in Theorem 3.1 an analytic map $\mu \mapsto \Phi(\mu)$ on a neighborhood $V_{1}$ of $\mu_{0}$, taking values in $\left(C^{\beta}\right)^{*}$, which corresponds for $\mu \in \mathcal{P}_{1}^{+}(F)$ to the integration against a $\mu$-stationary measure $v_{\mu}$ on $\partial_{B} \Gamma$.

Let $V=V_{0} \cap V_{1}$. For $\mu \in V \cap \mathcal{P}_{1}^{+}$, the integral expression (2.10) of the entropy gives

$$
\begin{aligned}
h(\mu) & =-\sum_{x \in F} \mu(x) \int_{\partial_{B} \Gamma} \log K_{\mu}^{B}\left(x^{-1}, \xi\right) \mathrm{d} v_{\mu}(\xi) \\
& =-\sum_{x \in F} \mu(x) \cdot \Phi(\mu)\left(\xi \mapsto \log f_{\mu}\left(x^{-1}, \xi\right)\right) .
\end{aligned}
$$

The expression on the second line is well defined and analytic on $V$, giving the desired analytic extension of the entropy. Indeed, by definition of $\mathcal{C}^{\beta}$, each function $\xi \mapsto f_{\mu}\left(x^{-1}, \xi\right)$ (with $x$ fixed) belongs to $C^{\beta}$ and depends analytically on $\mu$. Composing with the logarithm, applying the analytic linear form $\Phi(\mu)$, and doing a finite summation over $F$, everything remains analytic.

\section{Central limit theorem}

In this section, we prove the central limit theorem, Theorem 1.5. Once Proposition 3.6 is available, it follows using the method of Le Page [LP82] as described for instance in [BL85] or [BQ14]. Although the details are now standard, we will sketch the argument especially since the lack of true contraction on $\partial_{B}^{\prime} \Gamma$ creates possible periodicity problems. We will prove the result for measures with an exponential moment of order $\alpha>0$, as announced in Remark 1.6.

For $\mu \in \mathcal{P}_{1}^{+}(\alpha)$, we start from the operator $L_{\mu}$, acting on $C^{\beta}\left(\partial_{B}^{\prime} \Gamma\right)$ for some small enough $\beta>0$ by the formula $L_{\mu} u(\xi)=\sum_{g} \mu(g) u(g \xi)$. By Proposition 3.6, it has a simple eigenvalue at 1 . For $t \in \mathbb{R}$, define a perturbed operator $L_{\mu, t}$ by

$$
L_{\mu, t} u(\xi)=\sum_{g \in \Gamma} \mu(g) e^{\mathrm{i} t c_{B}(g, \xi)} u(g \xi),
$$

where $c_{B}$ is the Busemann cocycle (2.5). Then

$$
L_{\mu, t}^{2} u(\xi)=\sum_{g_{1}, g_{2}} \mu\left(g_{1}\right) \mu\left(g_{2}\right) e^{\mathbf{i} t c_{B}\left(g_{1}, g_{2} \xi\right)} e^{\mathbf{i} t c_{B}\left(g_{2}, \xi\right)} u\left(g_{1} g_{2} \xi\right)=\sum_{g} \mu^{* 2}(g) e^{\mathbf{i} t c_{B}(g, \xi)} u(g \xi),
$$

thanks to the cocycle equation (2.6) for $c_{B}$. Iterating this argument, one gets

$$
L_{\mu, t}^{n} u(\xi)=\sum_{g} \mu^{* n}(g) e^{\mathbf{i} t c_{B}(g, \xi)} u(g \xi) .
$$

In particular, if $Z_{n}$ denotes the right random walk driven by $\mu$, the characteristic function of $c_{B}\left(Z_{n}, \xi\right)$ can be expressed for all $\xi \in \partial_{B}^{\prime} \Gamma$ as follows:

$$
\mathbb{E}\left(e^{\mathbf{i} t c_{B}\left(Z_{n}, \xi\right)}\right)=L_{\mu, t}^{n} 1(\xi)
$$




\section{ANALYTICITY OF THE ENTROPY AND THE EsCAPE RATE}

Fix once and for all a point $\xi \in \partial_{B}^{\prime} \Gamma$. We will prove a central limit theorem for $c_{B}(\cdot, \xi)$, i.e., prove that $\left(c_{B}\left(Z_{n}, \xi\right)-n \ell\right) / \sqrt{n}$ converges in distribution to a Gaussian random variable. By Lévy's theorem, it suffices to prove the pointwise convergence of the characteristic functions. It will be obtained from (5.1) and the good spectral properties of $L_{\mu, t}$.

One checks easily that the map $t \mapsto L_{\mu, t}$ is analytic. By Proposition 3.6, the operator $L_{\mu, 0}=L_{\mu}$ has a simple eigenvalue at 1 , finitely many additional simple eigenvalues $\rho$ of modulus 1 , and the rest of its spectrum is contained in a disk of radius $<1$. This spectral picture persists for small $t$, see for instance [Kat66]: there are spectral projections $\Pi_{1, t}$ and $\Pi_{\rho, t}$ and $\Pi_{<1, t}$, all depending analytically on $t$, such that

$$
L_{\mu, t}^{n}=L_{\mu, t}^{n} \Pi_{1, t}+\sum_{\rho \neq 1} L_{\mu, t}^{n} \Pi_{\rho, t}+L_{\mu, t}^{n} \Pi_{<1, t} .
$$

(All these projections also depend on $\mu$, we suppress it from the notations for convenience.) Moreover, $\Pi_{1, t}$ is a one-dimensional projection, and $L_{\mu, t}$ acts on its image as the multiplication by the corresponding eigenvalue $\lambda(t)=\lambda_{\mu}(t)$. This eigenvalue also depends analytically on $t$.

Denote by $\ell$ the escape rate of the random walk. Then (5.1) implies that, for any $t$, and for large enough $n$ (so that $t / \sqrt{n}$ is in the neighborhood of 0 where the above spectral description holds true)

$$
\begin{aligned}
\mathbb{E}\left(e^{\mathrm{i} t \frac{c_{B}\left(Z_{n}, \xi\right)-n \ell}{\sqrt{n}}}\right) & =e^{-\mathbf{i} t \sqrt{n} \ell} L_{\mu, t / \sqrt{n}}^{n} 1(\xi) \\
= & e^{-\mathbf{i} t \sqrt{n} \ell}\left(\lambda(t / \sqrt{n})^{n} \Pi_{1, t / \sqrt{n}} 1(\xi)+\sum_{\rho \neq 1} L_{\mu, t / \sqrt{n}}^{n} \Pi_{\rho, t / \sqrt{n}} 1(\xi)+L_{\mu, t / \sqrt{n}}^{n} \Pi_{<1, t / \sqrt{n}} 1(\xi)\right) .
\end{aligned}
$$

Since the function 1 belongs to the eigenspace for the eigenvalue 1 , one has $\Pi_{\rho, 0} 1=\Pi_{<1,0} 1=0$. Hence, when $n$ tends to infinity, the functions $\Pi_{\rho, t / \sqrt{n}} 1$ and $\Pi_{<1, t / \sqrt{n}} 1$ tend to 0 in $C^{\beta}$, and therefore in $C^{0}$. As the iterates $L_{\mu, t / \sqrt{n}}^{n}$ are bounded in sup norm by 1 , it follows that $L_{\mu, t / \sqrt{n}}^{n} \Pi_{\rho, t / \sqrt{n}} 1(\xi)$ and $L_{\mu, t / \sqrt{n}}^{n} \Pi_{<1, t / \sqrt{n}} 1(\xi)$ tend to 0 .

In the same way, $\Pi_{1, t / \sqrt{n}} 1$ tends to $\Pi_{1,0} 1=1$. Finally, we obtain

$$
\mathbb{E}\left(e^{\mathbf{i} t \frac{c_{B}\left(Z_{n}, \xi\right)-n \ell}{\sqrt{n}}}\right)=e^{-\mathbf{i} t \sqrt{n} \ell} \lambda(t / \sqrt{n})^{n}+o(1) .
$$

As $\lambda$ is an analytic function with $\lambda(0)=1$, it has a Taylor expansion at 0 . It is convenient to write it as $\lambda(t)=e^{\mathbf{i} a t-b t^{2} / 2+o\left(t^{2}\right)}$, with $a=-\mathbf{i} \lambda^{\prime}(0)$ and $b=-a^{2}-\lambda^{\prime \prime}(0)$.

$>$ From this point on, two approaches are possible:

- One can proceed directly, without identifying $a$ and $b$.

- Or one can identify $a$ and $b$ by a tedious spectral computation.

The second approach is more precise (using it, one can prove that the variance in the central limit theorem is positive). However, since the first one is more illuminating, we will argue first in this way.

Reproducing the above computation but for $c_{B} / n$, one has

$$
\mathbb{E}\left(e^{\mathbf{i} t c_{B}\left(Z_{n}, \xi\right) / n}\right)=\lambda(t / n)^{n}+o(1) \rightarrow e^{\mathbf{i} a t} .
$$


By definition, $c_{B}\left(Z_{n}, \xi\right)=h_{\xi}\left(Z_{n}^{-1}\right)$. The point $Z_{n}^{-1}$ is distributed as the position $W_{n}$ at time $n$ of the reverse random walk, for the measure $\breve{\mu}$ given by $\check{\mu}(x)=\mu\left(x^{-1}\right)$. As $\check{\mu}$ is also admissible, this reverse random walk converges almost surely to a point on the Gromov boundary. As its exit distribution has no atom, this limit is almost surely different from $\pi_{B}(\xi)$, the projection of $\xi$ in the Gromov boundary. Then $h_{\xi}\left(W_{n}\right)-d\left(e, W_{n}\right)$ remains bounded almost surely by (2.3). Moreover, $d\left(e, W_{n}\right) / n$ tends to $\ell$, hence $h_{\xi}\left(W_{n}\right) / n$ tends almost surely to $\ell$. This implies that

$$
c_{B}\left(Z_{n}, \xi\right) / n=h_{\xi}\left(Z_{n}^{-1}\right) / n \text { tends in probability to } \ell .
$$

Therefore, $\mathbb{E}\left(e^{\mathrm{i} t c_{B}\left(Z_{n}, \xi\right) / n}\right)$ converges to $e^{\mathrm{i} t \ell}$. Hence, $a=\ell$.

For future use, note that the same argument shows that, for any sequence $r_{n}$ tending to infinity,

$$
\frac{c_{B}\left(Z_{n}, \xi\right)-d\left(e, Z_{n}\right)}{r_{n}} \text { tends in probability to } 0 .
$$

Using $a=\ell$, the equation (5.2) becomes

$$
\mathbb{E}\left(e^{\mathbf{i} t \frac{c_{B}\left(Z_{n}, \xi\right)-n \ell}{\sqrt{n}}}\right)=e^{-\mathbf{i} t \sqrt{n} \ell} e^{\mathbf{i} a \sqrt{n} t-b t^{2} / 2+o(1)}+o(1) \rightarrow e^{-b t^{2} / 2}
$$

We recall a version of Lévy's theorem: if a sequence of real random variables $X_{n}$ satisfies $\mathbb{E}\left(e^{\mathrm{i} t X_{n}}\right) \rightarrow$ $\varphi(t)$ where $\varphi$ is a continuous function, then $X_{n}$ converges in distribution to a random variable $X$, and $\varphi(t)=\mathbb{E}\left(e^{\mathbf{i} t X}\right)$. Hence, $\left(c_{B}\left(Z_{n}, \xi\right)-n \ell\right) / \sqrt{n}$ converges to a random variable, whose characteristic function is $e^{-b t^{2} / 2}$. This implies that $b \in[0, \infty)$. Writing it as $\sigma^{2} \geqslant 0$, we have proved that $\left(c_{B}\left(Z_{n}, \xi\right)-\right.$ $n \ell) / \sqrt{n}$ converges to $\mathcal{N}\left(0, \sigma^{2}\right)$ for any $\xi \in \partial_{B}^{\prime} \Gamma$.

Finally,

$$
\frac{d\left(e, Z_{n}\right)-n \ell}{\sqrt{n}}=\frac{c_{B}\left(Z_{n}, \xi\right)-n \ell}{\sqrt{n}}+\frac{d\left(e, Z_{n}\right)-c_{B}\left(Z_{n}, \xi\right)}{\sqrt{n}} .
$$

The first term on the right converges to $\mathcal{N}\left(0, \sigma^{2}\right)$. The second term on the right converges to 0 in probability by (5.3). Hence, their sum also converges to $\mathcal{N}\left(0, \sigma^{2}\right)$. This concludes the proof of the central limit theorem for $d\left(e, Z_{n}\right)$.

Let us show that $\sigma^{2}(\mu)$ depends analytically on $\mu$. One checks easily that the map $(\mu, t) \mapsto L_{\mu, t}$ is analytic. Hence, $(\mu, t) \mapsto \lambda_{\mu}(t)$ is also analytic, as simple isolated eigenvalues depend analytically on the operator, see [Kat66]. As a consequence, $\mu \mapsto \lambda_{\mu}^{\prime}(0)=\partial \lambda_{\mu}(t) /\left.\partial t\right|_{t=0}$ is analytic, and so is $\mu \mapsto \lambda_{\mu}^{\prime \prime}(0)$. Since $\ell(\mu)=a=-\mathbf{i} \lambda_{\mu}^{\prime}(0)$ and $\sigma^{2}(\mu)=b=-a^{2}-\lambda_{\mu}^{\prime \prime}(0)$, we recover simultaneously the analyticity of the escape rate stated in Theorem 1.1, and the analyticity of the variance in Theorem 1.5.

It remains to show that the variance $\sigma^{2}(\mu)$ is nonzero. For this, we need to identify $b$, by a spectral computation. Let $v$ denote the unique stationary measure of the random walk on $\partial_{B}^{\prime} \Gamma$, i.e., the fixed point of the operator $\left(L_{\mu}\right)^{*}$. Define $u_{t}=\Pi_{1, t} 1 / \int \Pi_{1, t} 1 \mathrm{~d} v$, it is an eigenfunction for the eigenvalue $\lambda(t)$ of $L_{\mu, t}$, normalized by $\int u_{t} \mathrm{~d} v=1$. Note that $\int \Pi_{1,0} 1 \mathrm{~d} v=\int 1 \mathrm{~d} v=1$, so that the denominator in the definition of $u_{t}$ does not vanish for small enough $t$. As $u_{t}$ depends analytically on $t$, we may write $u_{t}=u_{0}+\mathbf{i} t v+O\left(t^{2}\right)$, 
with $u_{0}=1$ and $\int v \mathrm{~d} v=0$ as $\int u_{t} \mathrm{~d} v=1$ for all $t$. Moreover, $L_{\mu, t} u_{t}=\lambda(t) u_{t}$. Keeping only the terms of order $\leqslant 1$ in this equation and using $\lambda(t)=1+\mathbf{i} t a+O\left(t^{2}\right)$, we get for all $\xi \in \partial_{B} \Gamma$

$$
\sum_{g} \mu(g) e^{\mathbf{i} t c_{B}(g, \xi)}(1+\mathbf{i} t v(g \xi))=(1+\mathbf{i} t a)(1+\mathbf{i} t v(\xi))+O\left(t^{2}\right)
$$

i.e.,

$$
1+\mathbf{i} t \sum_{g} \mu(g) c_{B}(g, \xi)+\mathbf{i} t \sum_{g} \mu(g) v(g \xi)=1+\mathbf{i} t a+\mathbf{i} t v(\xi)+O\left(t^{2}\right)
$$

Looking at the coefficient of $\mathbf{i} t$, we get

$$
\sum_{g} \mu(g)\left(c_{B}(g, \xi)+v(g \xi)-v(\xi)-a\right)=0 .
$$

Integrating this equation with respect to $v$ and using its stationarity, we recover the equality

$$
a=\int c_{B}(g, \xi) \mathrm{d} \mu(g) \mathrm{d} v(\xi)=\ell
$$

that we proved before by a probabilistic argument.

Define a new cocycle

$$
\tilde{c}(g, \xi)=c_{B}(g, \xi)+v(g \xi)-v(\xi)-\ell
$$

It satisfies $\sum \mu(g) \tilde{c}(g, \xi)=0$ for all $\xi$ by (5.4). Moreover, $c_{B}\left(Z_{n}, \xi\right)-n \ell-\tilde{c}\left(Z_{n}, \xi\right)=v(\xi)-v\left(Z_{n} \xi\right)$ is uniformly bounded, hence it is equivalent to have the central limit theorem for $c_{B}$ or for $\tilde{c}$, and the asymptotic variances coincide.

Let us use $\tilde{c}$ instead of $c_{B}$, to define a new operator $\tilde{L}_{t}$ by $\tilde{L}_{t} u(\xi)=\sum_{g} \mu(g) e^{\mathbf{i} t \tilde{c}(g, \xi)} u(g \xi)$. All the above discussion applies to this operator. We get in particular a new eigenvalue $\tilde{\lambda}(t)$, a new eigenfunction $\tilde{u}_{t}$ which can be expanded as $1+\mathbf{i} t \tilde{v}+O\left(t^{2}\right)$ with $\int \tilde{v} \mathrm{~d} v=0$, a new value of the derivative $\tilde{a}$ of $\tilde{\lambda}$ at 0 . The equation (5.4) becomes

$$
\sum_{g} \mu(g)(\tilde{c}(g, \xi)+\tilde{v}(g \xi)-\tilde{v}(\xi)-\tilde{a})=0
$$

By construction, $\sum \mu(g) \tilde{c}(g, \xi)=0$ for all $g$. Integrating the above equation with respect to $v$, we get $\tilde{a}=0$. Then (5.5) yields $\tilde{v}=L_{0} \tilde{v}$. By Proposition 3.6, $\tilde{v}$ is constant. As its integral is zero, $\tilde{v}=0$. Finally, $\tilde{u}_{t}=1+O\left(t^{2}\right)$.

We can now compute the second term in the expansion of $\tilde{\lambda}(t)$. We have

$$
\begin{aligned}
\tilde{\lambda}(t) & =\int \tilde{\lambda}(t) \tilde{u}_{t} \mathrm{~d} v=\int \tilde{L}_{t} \tilde{u}_{t} \mathrm{~d} v=\int \tilde{L}_{t}\left(\tilde{u}_{t}-1\right) \mathrm{d} v+\int \tilde{L}_{t} 1 \mathrm{~d} v \\
& =\int\left(\tilde{L}_{t}-L_{0}\right)\left(\tilde{u}_{t}-1\right) \mathrm{d} v+\int \tilde{L}_{t} 1 \mathrm{~d} v .
\end{aligned}
$$

The first term is $O\left(t^{3}\right)$ as $\tilde{u}_{t}-1=O\left(t^{2}\right)$ and $\tilde{L}_{t}-L_{0}=O(t)$. The second term is equal to

$$
\int e^{\mathrm{i} t \tilde{c}(g, \xi)} \mathrm{d} \mu(g) \mathrm{d} v(\xi)=1+\mathbf{i} t \int \tilde{c}(g, \xi) \mathrm{d} \mu(g) \mathrm{d} v(\xi)-\frac{t^{2}}{2} \int \tilde{c}(g, \xi)^{2} \mathrm{~d} \mu(g) \mathrm{d} v(\xi)+O\left(t^{3}\right) .
$$


Since the variance in the central limit theorem is given by $-\tilde{\lambda}^{\prime \prime}(0)$, we obtain

$$
\sigma^{2}=\sum_{g} \mu(g) \int \tilde{c}(g, \xi)^{2} \mathrm{~d} v(\xi) \geqslant 0
$$

If it vanishes, then all quantities $\tilde{c}(g, \xi)$ are zero for $g$ in the support of $\mu$. Using the definition of $\tilde{c}$ and the cocycle equation for $c_{B}$, this gives

$$
c_{B}\left(Z_{n}, \xi\right)=n \ell+v(\xi)-v\left(Z_{n} \xi\right)
$$

for any $Z_{n}$ in the support of $\mu^{* n}$. Take $n$ with $\mu^{* n}(e)>0$, by (3.9). Applying the above equation to $Z_{n}=e$, we get $0=n \ell$, a contradiction with the positivity of $\ell$ that follows from the non-amenability of $\Gamma$. Thus, $\sigma^{2}>0$.

Remark 5.1. Several of the above arguments could be replaced by martingale arguments, as in [BQ16], but we have opted for a self-contained spectral treatment. Note that many objects that appear in this proof are also present in the martingale argument. For instance, the key step in the martingale argument is to construct a function $v$ such that (5.4) holds. This function $v$ (a solution of the Poisson equation) appears naturally in the spectral framework, as the derivative of $t \mapsto u_{t}$.

There is nothing special about the Busemann cocycle in the above proof: the next theorem is proved exactly in the same way.

Theorem 5.2. Let $\Gamma$ be a nonelementary hyperbolic group with a word distance, endowed with an admissible probability measure $\mu$ having an exponential moment. Let $c: \Gamma \times \partial_{B}^{\prime} \Gamma \rightarrow \mathbb{R}$ be a cocycle on a minimal subset $\partial_{B}^{\prime} \Gamma$ of the Busemann boundary $\partial_{B} \Gamma$. Assume that the cocycle is Hölder continuous, i.e., for every $g \in \Gamma$, the map $\xi \mapsto c(g, \xi)$ is Hölder continuous on $\partial_{B}^{\prime} \Gamma$.

Let $Z_{n}$ denote the right random walk on $\Gamma$ driven by $\mu$. Then there exists $\ell \in \mathbb{R}$ such that, for any $\xi \in \partial_{B}^{\prime} \Gamma$, the quantity $c\left(Z_{n}, \xi\right) / n$ converges almost surely to $\ell$. Moreover, there exists $\sigma^{2} \geqslant 0$ such that

$$
\frac{c\left(Z_{n}, \xi\right)-n \ell}{\sqrt{n}} \rightarrow \mathcal{N}\left(0, \sigma^{2}\right)
$$

Additionally, $\sigma^{2}=0$ if and only if there exists a Hölder continuous function $v$ on $\partial_{B}^{\prime} \Gamma$ such that $c(g, \xi)=$ $v(g \xi)-v(\xi)$ for all $g \in \Gamma$ and all $\xi \in \partial_{B}^{\prime} \Gamma$ (and in this case $\ell=0$ also). This is also equivalent to the uniform boundedness of $c$.

Finally, both $\ell$ and $\sigma$ depend analytically on $\mu$.

\section{References}

[Anc88] Alano Ancona, Positive harmonic functions and hyperbolicity, Potential theory, surveys and problems (Prague, 1987), Lecture Notes in Math., vol. 1344, Springer, Berlin, 1988, pp. 1-23. MR MR973878 8

[Bjö10] Michael Björklund, Central limit theorems for Gromov hyperbolic groups, J. Theoret. Probab. 23 (2010), 871-887. MR MR2679960 5, 11 
[BL85] Philippe Bougerol and Jean Lacroix, Products of random matrices with applications to Schrödinger operators, Progress in Probability and Statistics, vol. 8, Birkhäuser Boston Inc., Boston, MA, 1985. MR MR886674 30

[BQ14] Yves Benoist and Jean-François Quint, Random walks on reductive groups, preprint, 2014. $11,13,14,30$

[BQ16] Central limit theorem on hyperbolic groups, Izv. Ross. Akad. Nauk Ser. Mat. 80 (2016), no. 1, 5-26. MR 3462675 5, 34

[CP01] Michel Coornaert and Athanase Papadopoulos, Horofunctions and symbolic dynamics on Gromov hyperbolic groups, Glasg. Math. J. 43 (2001), 425-456. MR MR1878587 6, 12

[EK13] Anna Erschler and Vadim Kaimanovich, Continuity of asymptotic characteristics for random walks on hyperbolic groups, Funktsional. Anal. i Prilozhen. 47 (2013), 84-89. MR MR3113872 2

[GdlH90] Étienne Ghys and Pierre de la Harpe (eds.), Sur les groupes hyperboliques d'après Mikhael Gromov, Progress in Mathematics, vol. 83, Birkhäuser Boston Inc., Boston, MA, 1990, Papers from the Swiss Seminar on Hyperbolic Groups held in Bern, 1988. MR MR1086648 5

[Gil07] Lorenz A. Gilch, Rate of escape of random walks on free products, J. Aust. Math. Soc. 83 (2007), 31-54. MR MR2378433 2

[Gil08]___ Rate of escape of random walks on regular languages and free products by amalgamation of finite groups, Fifth Colloquium on Mathematics and Computer Science, Discrete Math. Theor. Comput. Sci. Proc., AI, Assoc. Discrete Math. Theor. Comput. Sci., Nancy, 2008, pp. 405-420. MR MR2508803 2

[Gil11] Asymptotic entropy of random walks on free products, Electron. J. Probab. 16 (2011), no. 3, 76-105. MR MR2749773 2

[GL13a] Lorenz Gilch and François Ledrappier, Regularity of the drift and entropy of random walks on groups, Publ. Mat. Urug. 14 (2013), 147-158. MR MR3235351 2, 4

[GL13b] Sébastien Gouëzel and Steven P. Lalley, Random walks on co-compact Fuchsian groups, Ann. Sci. Éc. Norm. Supér. (4) 46 (2013), 129-173 (2013). MR MR3087391 18, 23

[GMM15] Sébastien Gouëzel, Frédéric Mathéus, and François Maucourant, Entropy and drift in word hyperbolic groups, Preprint, 2015. 3, 5, 8

[Gou15] Sébastien Gouëzel, Martin boundary of random walks with unbounded jumps in hyperbolic groups, Ann. Probab. 43 (2015), 2374-2404. MR MR3395464 5

[Haï13] Peter Haïssinsky, Marches aléatoires sur les groupes hyperboliques, Géométrie ergodique, Monogr. Enseign. Math., vol. 43, Enseignement Math., Geneva, 2013, pp. 199-265. MR MR3220556 2, 5 
[Hen93] Hubert Hennion, Sur un théorème spectral et son application aux noyaux lipchitziens, Proc. Amer. Math. Soc. 118 (1993), 627-634. MR MR1129880 15

[HMM16] Peter Haïssinsky, Pierre Mathieu, and Sebastian Müller, Renewal theory for random walks on surface groups, Ergodic Theory Dynam. Systems (2016), 1-25. 2, 5

[HPS77] Morris W. Hirsch, Charles C. Pugh, and Michael Shub, Invariant manifolds, Lecture Notes in Mathematics, Vol. 583, Springer-Verlag, Berlin, 1977. MR MR0501173 3

[Kai00] Vadim A. Kaimanovich, The Poisson formula for groups with hyperbolic properties, Ann. of Math. (2) 152 (2000), 659-692. MR MR1815698 7

[Kat66] Tosio Kato, Perturbation theory for linear operators, Die Grundlehren der mathematischen Wissenschaften, Band 132, Springer-Verlag New York, Inc., New York, 1966. MR MR0203473 15, 16, 31, 32

[KB02] Ilya Kapovich and Nadia Benakli, Boundaries of hyperbolic groups, Combinatorial and geometric group theory (New York, 2000/Hoboken, NJ, 2001), Contemp. Math., vol. 296, Amer. Math. Soc., Providence, RI, 2002, pp. 39-93. MR MR1921706 6

[KV83] V. A. Kaŭmanovich and A. M. Vershik, Random walks on discrete groups: boundary and entropy, Ann. Probab. 11 (1983), 457-490. MR MR704539 3, 8

[Led12] François Ledrappier, Analyticity of the entropy for some random walks, Groups Geom. Dyn. 6 (2012), 317-333. MR MR2914862 2

[Led13]__ Regularity of the entropy for random walks on hyperbolic groups, Ann. Probab. 41 (2013), 3582-3605. MR MR3127892 3, 5

[LP82] Émile Le Page, Théorèmes limites pour les produits de matrices aléatoires, Probability measures on groups (Oberwolfach, 1981), Lecture Notes in Math., vol. 928, Springer, BerlinNew York, 1982, pp. 258-303. MR 669072 4, 11, 13, 30

[Mat15] Pierre Mathieu, Differentiating the entropy of random walks on hyperbolic groups, Ann. Probab. 43 (2015), 166-187. MR MR3298471 3

[MS16] Pierre Mathieu and Alessandro Sisto, Deviation inequalities and CLT for random walks on acylindrically hyperbolic groups, preprint, 2016. 5

[Muj86] Jorge Mujica, Complex analysis in Banach spaces, North-Holland Mathematics Studies, vol. 120, North-Holland Publishing Co., Amsterdam, 1986. MR MR842435 9

[Saw97] Stanley A. Sawyer, Martin boundaries and random walks, Harmonic functions on trees and buildings (New York, 1995), Contemp. Math., vol. 206, Amer. Math. Soc., Providence, RI, 1997, pp. 17-44. MR MR1463727 8 


\section{AUTHOR}

\section{Sébastien Gouëzel}

Laboratoire Jean Leray, CNRS UMR 6629

Université de Nantes, 2 rue de la Houssinière

44322 Nantes, France

sebastien.gouezel@univ-nantes.fr 